\title{
Femtosecond Control of the Magnetization in Ferromagnetic Semiconductors
}

\author{
J. Chovan and I. E. Perakis \\ Institute of Electronic Structure $\&$ Laser, Foundation for Research and \\ Technology-Hellas and Department of Physics, University of Crete, Heraklion, Greece
}

(Dated: November 6, 2018)

\begin{abstract}
We develop a theory of collective spin dynamics triggered by ultrafast optical excitation of ferromagnetic semiconductors. Using the density matrix equations of motion in the mean field approximation and including magnetic anisotropy and hole spin dephasing effects, we predict the development of a light-induced magnetization tilt during ultra-short time intervals comparable to the pulse duration. This femtosecond dynamics in the coherent temporal regime is governed by the interband nonlinear optical polarizations and is followed by a second temporal regime governed by the magnetic anisotropy of the Fermi sea. We interpret our numerical results by deriving a Landau-Gilbert-like equation for the collective spin, which demonstrates an ultrafast correction to the magnetic anisotropy effective field due to second order coherent nonlinear optical processes. Using the Lindblad semigroup method, we also derive a contribution to the interband polarization dephasing determined by the Mn spin and the hole spin dephasing. Our predicted magnetization tilt and subsequent nonlinear dynamics due to the magnetic anisotropy can be controlled by varying the optical pulse intensity, duration, and helicity and can be observed with pump-probe magneto-optical spectroscopy.
\end{abstract}

PACS numbers: 78.47.J-, 78.20.Ls, 78.30.Fs, 42.50.Md

\section{INTRODUCTION}

The interaction between itinerant carrier spins and localized magnetic moments leads to carrier-mediated ferromagnetic order in a wide variety of systems, ranging from ferromagnetic semiconductors such as $\mathrm{EuO}$, EuS, chrome spinels, or pyrochlore $\frac{1}{2}$ to manganese oxides (manganites) ${ }^{2}$ and III-Mn-V ferromagnetic semiconductors $3.4,5$. Such materials offer potential for novel spintronics applications $\mathbf{6}^{6}$. Their magnetic and transport properties are intimately related and can be controlled by varying e.g. the carrier density, spin, and distribution.

The non-equilibrium and dynamical properties of ferromagnetic semiconductors and magnetically-ordered systems are currently under investigation. For THz spintronic and magnetic devices, ultrafast information storage, recovery, and processing is required, e.g. the development of devices with sub-picosecond readout times of the magnetic states. This goal requires femtosecond spin manipulation and control. The physical processes that govern the magnetization dynamics during timescales shorter than the characteristic response times of the magnetic system are still under debate. During such timescales, the validity of conventional thermodynamic concepts for describing a magnetic system become questionable. Ultrafast pump-probe magnetooptical spectroscopy can shed light into this fundamental problem. In these experiments, an ultra-short pump optical pulse excites optical polarizations, non-thermal populations, and carrier spins, which then trigger a magnetization dynamics measured as function of time (Faraday or Kerr rotation) $)^{7}$.

To interpret such experiments, it is useful to distinguish between different stages of time evolution of the photoexcited system. During the initial sub-picosecond regime, shorter than the dephasing times or optical pulse duration, the dynamics of the collective magnetization is triggered by optical polarizations and photoexcited carrier spins. The response of the magnetic system is controlled by the nonlinear optical excitation and conventional thermodynamic concepts do not apply. In this initial regime, magnetic anisotropy can play a role by affecting the photoexcited carrier spin. After the pulse is gone, the light-induced quantum mechanical coherences decay and the photoexcited carriers relax by interacting with the hole Fermi sea. The carrier temperature is thus elevated above the lattice temperature within hundreds of femtoseconds. The hole population is eventually described by a hot Fermi-Dirac distribution, which transfers its excess energy to the lattice within a few picoseconds. At the same time, the magnetic axes of the system change, due to e.g. the transient temperature elevation. Such quasi-thermal changes in the magnetic anisotropy are due to the Fermi sea carriers and should be contrasted to the magnetic anisotropy contribution of the non-thermal photoexcited carriers in the initial temporal regime. After the equilibration of the carrier and lattice systems, their common temperature relaxes via a slow (nanosecond) thermal diffusion process, which returns the magnetic system to its equilibrium configuration. In this paper we discuss a mechanism for coherent non-thermal magnetization manipulation and neglect all thermal effects due to elevated carrier and spin temperatures.

Most of the ultrafast magneto-optical experiments performed so far in magnetic metals, insulators, and semiconductors observed magnetization dynamics that could be interpreted in terms of light-induced timedependent thermal effects. Following the observation of Ref [8, many works focussed on ultrafast light-induced 
demagnetization, which involves the time-dependent collective magnetization amplitude. The physical mechanisms that lead to quenching of the magnetization within a picosecond or less in materials ranging from transition metals to III ( Mn)V semiconductors are still under debate, but are mostly believed to be triggered by transient changes in the carrier and spin effective temperatures $, 7,8,9,10,11,12,13,14$ However, transient magnetic effects have also been observed in the initial nonthermal temporal regime, where the concept of carrier temperature is not meaningful. $\frac{9,10}{}$ Light-induced changes in the magnetization orientation have also been observed in both metals and semiconductors and mostly attributed to transient changes in the magnetic easy axes due to temperature elevation. $15,16,17,18,19,20,21$ Such thermal quasi-equilibrium effects induce a magnetization precession with a period $\sim 100$ ps.

In most of the above experiments, the observed magnetization changes result from the transient temperature rise following optical absorption and carrier relaxation. However, there is a limit in the magnetization speed that can arise from incoherent processes largely based on the heating of the magnetic system. Far more desirable is magnetization control based on coherent and non-thermal physical processes. The femtosecond coherent temporal regime offers the most flexibility for fast magnetization control limited only by the optical pulse duration. Coherent manipulation of the magnetization was demontrated experimentally in magnetic dielectrics and insulators $22,23,24$, while the interplay of coherent excitation and spin-orbit interaction in magnetic insulators was addressed theoretically in Refs. 25,26.

Compared to other magnetically-ordered materials, III-Mn-V ferromagnetic semiconductors offer certain advantages and new possibilities for ultafast magnetization control and dynamics. These advantages stem from the carrier-induced nature of the magnetic order and the clear distinction between localized (Mn) and itinerant (valence band hole) spins. Static measurements have shown that III-Mn-V heterostructures are highly sensitive to external stimuli such as electrical gate, currents, or light $27,28,29,30$ A light-induced out-of-plane magnetization rotation towards the direction of propagation of a circularly polarized optical field perpendicular to the ground state magnetization was reported in Refs. 31,32 for $\mathrm{Ga}(\mathrm{Mn})$ As epilayers. Later ultrafast experiments interpreted their findings in terms of photoexcited carrier spin $^{33}$ and thermal ${ }^{13,14}$ effects (for a review see Ref. 7).

Recently, Wang et.al. ${ }^{34}$ reported an enhancement of the magnetization amplitude and the ferromagnetic order in GaMnAs induced by the photoexcited hole population. This enhancement occurs on a $\sim 100$ ps time scale, following the initial subpicosecond demagnetization ${ }^{14}$ and thermalization. More recently, Wang et.al. ${ }^{35}$ reported the first observation of two temporal regimes of magnetization dynamics in III-Mn-V semiconductors. The first regime lasts for a few hundreds of fs and is governed by a quasi-instantaneous tilt of the collective mag- netization in response to optical excitation at high energies $(\sim 3.1 \mathrm{eV})$. In this femtosecond regime, a photoinduced four-state ferromagnetic hysterisis was measured, which implies femtosecond detection of magnetic memory states. This initial magnetization dynamics is clearly distinguished from the subsequent thermal regime, which is governed by magnetization precession on the 100ps timescale around the magnetic easy axes.

The observations of Ref 35 point out the need for a microscopic theory of collective spin dynamics in the initial coherent regime of $\mathrm{III}(\mathrm{Mn}) \mathrm{V}$ semiconductors, which treats the nonlinear response of the magnetization to the ultrafast optical excitation. The theoretical prediction of a light-induced magnetic interaction resulting in a Kondo resonance in the nonlinear optical response and the pump-probe spectra of doped semiconductors was reported in Ref 36 . This light-induced many-body effect is generated by a second order Raman-like process and should be most pronounced in the case of belowresonance photoexcitation in the transparency regime, where heating effects are suppressed. Ref. 37 suggested the possibility of inducing ferromagnetic order by exciting undoped paramagnetic II-Mn-VI semiconductors well below the optical absorption threshold. A brief description of a microscopic mechanism for coherent ultrafast magnetization dynamics in III-Mn- $\mathrm{V}$ ferromagnetic semiconductors was presented in Ref. 38, while Ref. 39 addressed the subsequent incoherent regime and attributed the ultrafast demagnetization to the scattering of the Mn spins with spin-flip excitations of the hot hole Fermi sea.

In this paper we develop in detail a theory that describes the ultrafast nonlinear response of the collective spins in ferromagnetic semiconductors. We present calculations for the most basic Hamiltonian that applies to a wide range of ferromagnetic semiconductor systems. The magnetic exchange interaction and the coupling to the optical field are treated within the mean field approximation, which has been shown to describe well the ground state and thermodynamic properties of III-Mn-V semiconductors $\frac{4,5}{5}$ as well as the ultarafast nonlinear optical response of semiconductors for strong photoexcitation $\stackrel{40,41}{ }$ In Section III we set up the problem at hand, while in Section III we derive the mean field equations of motion in the coherent limit in the case of several coupled valence bands. The dephasing effects due to the mixing of the hole spin states are described in Section IV with the Lindblad semigroup method. ${ }^{2}$ This method allows us to treat consistently both hole spin relaxation/dephasing and interband polarization dephasing resulting from hole spin-flip interactions. In Section V] we extract a simple one-band model from the full theory and use it in SectionVI to calculate the light-induced Mn spin trajectories and discuss the role of hole spin and interband polarization dephasing, magnetic anisotropy, and photoexcitation intensity and duration. Our numerical results predict an ultrafast tilt of the Mn spin away from its initial (equilibrium) value, which develops on a timescale comparable to the optical pulse duration. 
The magnitude of this tilt is controlled by the optical pulse intensity and duration. The direction of the tilt and overall shape of the Mn spin trajectory depend sensitively on the hole spin dephasing and on the interband polarizations. In Section VII we interpret the above numerical results by deriving from the full theory an effective Landau-Gilbert-like 43 equation of motion for the Mn spin using the adiabatic following approximation. We show that the effective magnetic anisotropy fields that govern the precession and relaxation within the above Landau-Gilbert picture acquire a time-dependent correction determined by the optical pulse amplitude, the photoexcited interband polarizations, and the mixing of the hole spin states. This correction results in magnetization dynamics on a timescale comparable to the optical pulse duration and may be interpeted in terms of light-induced precession and relaxation. After the pulse is gone, the Mn spin trajectory is controlled by the magnetic anisotropies due to the Fermi sea (thermal) carriers. If the initial magnetization response to the optical excitation results in a sufficiently small tilt, the Mn spin precesses around its initial configuration, with a period determined by the zero-momentum magnon energy, as described by linearizing the equations of motion. On the other hand, for sufficiently high photoexcitation intensity, the initial light-induced magnetization tilt brings the magnetic system sufficiently far from the magnetic energy minimum so that nonlinear magnetic effects become important. In this case, the Mn spin evolution differs from a simple precession. Our theory predicts two distinct temporal regimes of magnetization evolution, the first of which is governed by the optical excitation while the second is governed by the magnetic anisotropies due to the thermal carriers. We discuss the signatures of the above transient and nonlinear magnetic effects in ultrafast magneto-optical pump-probe spectroscopy and end with our conclusions in Section VIII. The details of our calculations are presented in the five Appendices.

\section{PROBLEM SETUP}

We start with the Hamiltonian ${ }^{4,5} H(t)=H+H_{L}(t)$, where $H=K_{e}+K_{h}+H_{\text {exch }}$ is the Hamiltonian in the absence of optical excitation and $H_{L}(t)$ describes the coupling of the optical fields.

$$
K_{e}=\sum_{\mathbf{k} n} \varepsilon_{\mathbf{k} n}^{c} \hat{e}_{\mathbf{k} n}^{\dagger} \hat{e}_{\mathbf{k} n}, K_{h}=\sum_{\mathbf{k} n} \varepsilon_{-\mathbf{k} n}^{v} \hat{h}_{-\mathbf{k} n}^{\dagger} \hat{h}_{-\mathbf{k} n}
$$

describe the electron and hole band energies. The conduction band electrons are created by the operator $\hat{e}_{\mathbf{k} n}^{\dagger}$, where $\mathbf{k}$ is the momentum and $n$ labels different conduction bands. $\varepsilon_{\mathbf{k} n}^{c}$ is the $n$-band dispersion (we set $\hbar=1$ ). The valence holes are created by the operator $\hat{h}_{\mathbf{k} n}^{\dagger}$, where $\mathbf{k}$ is the hole momentum and $n$ the band index. Their dispersion $\varepsilon_{\mathbf{k} n}^{v}$ is determined by the bandstructure. The Mn impurities act as acceptors, which create a hole Fermi sea in the valence band, and also provide randomly distributed $S=5 / 2$ spins, $\mathbf{S}_{j}$, that are localized at positions $\mathbf{R}_{j}$. These local moments interact with the hole spin via the antiferromagnetic Kondo-like exchange interaction

$$
H_{\mathrm{exch}}=\frac{\beta}{V} \sum_{j \mathbf{k} \mathbf{k}^{\prime} n n^{\prime}} \mathbf{S}_{j} \cdot \mathbf{s}_{n n^{\prime}} e^{i\left(\mathbf{k}-\mathbf{k}^{\prime}\right) \cdot \mathbf{R}_{j}} \hat{h}_{-\mathbf{k} n}^{\dagger} \hat{h}_{-\mathbf{k}^{\prime} n^{\prime}}
$$

where $\mathbf{s}$ is the hole spin operator, $V$ is the volume, and $\beta$ is the exchange constant. Finally, the coupling of the optical pulse is described by

$$
H_{L}(t)=-\sum_{n n^{\prime} \mathbf{k}} d_{n n^{\prime}}(t) \hat{e}_{\mathbf{k} n}^{\dagger} \hat{h}_{-\mathbf{k} n^{\prime}}^{\dagger}+h . c,
$$

where $d_{n n^{\prime}}(t)=\mu_{n n^{\prime}} \mathcal{E}(t)$ is the Rabi energy, $\mathcal{E}(t)=$ $\mathcal{E} \exp \left[-t^{2} / \tau_{p}^{2}\right]$ is the optical (pump) pulse, with duration $\tau_{p}$, and $\mu_{n n^{\prime}}$ is the dipole transition matrix element between the valence band $n^{\prime}$ and the conduction band $n$. The optical pulse propagates along the growth direction $\mathrm{z}$, which is perpendicular to the ground state spins.

The ground state, thermodynamic, and transport properties of III $(\mathrm{Mn}) \mathrm{V}$ semiconductors in the metallic regime (hole densities $\sim 10^{20} \mathrm{~cm}^{-3}$ ) are well described by treating the magnetic exchange interaction, Eq.(2), within the mean field virtual crystal approximation. $\underline{5}$ This approximation neglects spatial correlations and assumes uniformly distributed classical Mn spins, justified in the limit $S \rightarrow \infty$, where $S$ is the Mn spin amplitude. The holes then experience an effective magnetic field proportional to the Mn spin. The valence band splits into two spin-polarized bands separated by the magnetic exchange energy $\Delta=\beta c S$, where $c$ is the density of the localized Mn spins. The typical values of $\Delta$ observed in the GaMnAs and InMnAs ferromagnetic semiconductors that exhibit the highest critical temperatures are comparable to the Fermi energy, $E_{F} \sim 100 \mathrm{meV}$, of the ground state hole Fermi sea.

In the ground state, the Mn spin points along the easy axis direction in order to minimize the total energy of the Fermi sea carriers:

$$
E_{h}(\mathbf{S})=\sum_{\mathbf{k} i} E_{\mathbf{k} i}^{h} n_{\mathbf{k} i}
$$

where $E_{\mathbf{k} i}^{h}(\mathbf{S})$ are the eigensvalues of the Hamiltonian $K_{h}+H_{\text {exch }}$ for given Mn spin $\mathbf{S}$ and $n_{\mathbf{k} i}$ are the populations of the corresponding eigenstates. In III-Mn-V semiconductors, this mean field total energy depends strongly on the orientation of $\mathbf{S} .44,45$ This anisotropy is believed to mainly arise from the valence bandstructure, in particular the spin-orbit coupling of different valence bands, which was taken into account in Refs, 44, 45 within the $\mathbf{k} \cdot \mathbf{p}$ envelope function approximation. The easy axis depends sensitively on the hole distribution among different valence bands. The calculated $E_{h}(\mathbf{S})$ is well described by an expansion in terms of $\mathbf{S}^{44}$ The following expansion describes the static experimental measurements: ${ }^{46}$

$$
E_{h}=K_{c}\left(\hat{S}_{x}^{2} \hat{S}_{y}^{2}+\hat{S}_{x}^{2} \hat{S}_{z}^{2}+\hat{S}_{y}^{2} \hat{S}_{z}^{2}\right)+K_{u} \hat{S}_{x}^{2}+K_{u z} \hat{S}_{z}^{2},
$$


where $\hat{\mathbf{S}}=\mathbf{S} / S, K_{c}$ is the lowest cubic anisotropy constant, and $K_{u}$ and $K_{u z}$ are the first order uniaxial anisotropy constants, attributed to strain, whose origin is still being debated. 47 For $K_{u}=K_{u z}=0$, the ground state magnetization is either parallel to the axes $\pm x, \pm y$ or $\pm z$, if $K_{c}>0$, or points along the diagonals $|x|=|y|=|z|$, if $K_{c}<0$. For the parameters of interest in $\mathrm{Ga}(\mathrm{Mn})$ As epilayers, $K_{c}<0$, and the easy axis direction is determined by the magnitude and sign of the uniaxial anisotropy constants $K_{u}$ and $K_{u z}$. A sufficiently large $K_{u z}>0$ ensures that the ground state magnetization lies within the $x-y$ plane as observed experimentally. The observed temperature and hole concentration dependence of the easy axis suggests that $K_{u}>0.46$ For $K_{u}>\left|K_{c}\right|$, the easy axis points along the $\pm y$ axis, while for $K_{u}<\left|K_{c}\right|$ it points at an angle $\phi$ from the $x$-axis, where $\cos 2 \phi=K_{u} /\left|K_{c}\right|$.

Even though $\mathbf{k} \cdot \mathbf{p}$ and mean field theory explain the main anisotropy effects, the interpretation of the observed changes of the in-plane easy axis with temperature and hole concentration require further theoretical investigations. A complete microscopic theory that includes the magnetic anisotropy becomes even more complicated in the case of ultrafast optical excitation. As observed experimentally, 35 the magnetization responds to the photoexcitation of high energy $(\sim 3.1 \mathrm{eV})$ valence bands states, which are empty in the ground state and lie far from the Brillouin zone center, well before carrier or spin thermalization. In this initial highly nonequilibrium regime, a fully microscopic theory must address both the valence bandstructure at $\sim 3 \mathrm{eV}$ energies and the coherent and non-thermal effects. Here we address the general magnetic semiconductor system and include the magnetic anisotropy effects by using the Fermi sea energy expression Eq.(5). The time dependent response of the Mn spin $\mathbf{S}$ to the nonlinear optical excitation, discussed in the next section, changes $E_{h}(\mathbf{S})$, which leads to an additional complex nonlinear magnetization dynamics.

\section{EQUATIONS OF MOTION}

To describe the ultrafast optical and spin response, we proceed in two steps. In this section we derive the mean field equations of motion, while in the next section we derive the dephasing contributions due to the hole spinflip interactions. The components of the density matrix $\langle\rho\rangle$ are obtained from the equations of motion

$$
i \partial_{t}\langle\rho\rangle=\left.\langle[\rho, H(t)]\rangle\right|_{H F}+\left.i \partial_{t}\langle\rho\rangle\right|_{\text {relax }},
$$

where the last term describes the dephasing and relaxation contributions. By factorizing all higher density matrices (Hartree-Fock approximation), which couple due to the many-body exchange interaction, we obtain a closed system of equations for the optical polarizations, spins, and carrier populations and coherences.

We are interested in calculating the macroscopic magnetization measured in ultrafast pump-probe magneto- optical experiments. This is dominated by the contribution of the average Mn spin

$$
\mathbf{S}=\frac{1}{c V} \sum_{i}\left\langle\mathbf{S}_{i}\right\rangle
$$

From Eq.(6) we obtain within the mean field approximation the equation of motion that describes the magnetization dynamics:

$$
\partial_{t} \mathbf{S}=\mathbf{S} \times\left[\mathbf{H}-\frac{\beta}{V} \sum_{\mathbf{k}} \mathbf{s}_{\mathbf{k}}^{h}\right]
$$

where $\mathbf{H}$ is a magnetic field. The right hand side (rhs) of the above equation describes the precession of the Mn spin around an effective time-dependent magnetic field determined by $\mathbf{H}$ and the mean hole spin

$$
\mathbf{s}_{\mathbf{k}}^{h}=\sum_{n n^{\prime}} \mathbf{s}_{n n^{\prime}}\left\langle\hat{h}_{-\mathbf{k} n}^{\dagger} \hat{h}_{-\mathbf{k} n^{\prime}}\right\rangle
$$

where $\mathbf{s}_{n n^{\prime}}$ are the matrix elements of the hole spin operator $\mathbf{s}$ between the valence band eigenstates. Eq. (8) corresponds to the Landau-Gilbert picture of magnetization dynamics ${ }^{43}$ and conserves the amplitude of $\mathbf{S}$.

The hole spin, populations, and inter-valence band coherences are described by the density matrices $\left\langle\hat{h}_{-\mathbf{k} n}^{\dagger} \hat{h}_{-\mathbf{k} n^{\prime}}\right\rangle$, whose equations of motion read

$$
\begin{aligned}
& i \partial_{t}\left\langle\hat{h}_{-\mathbf{k} n}^{\dagger} \hat{h}_{-\mathbf{k} n^{\prime}}\right\rangle=\left(\varepsilon_{\mathbf{k} n^{\prime}}^{v}-\varepsilon_{\mathbf{k} n}^{v}\right)\left\langle\hat{h}_{-\mathbf{k} n}^{\dagger} \hat{h}_{-\mathbf{k} n^{\prime}}\right\rangle \\
& +\beta c \sum_{m^{\prime}} \mathbf{S} \cdot\left[\mathbf{s}_{n^{\prime} m^{\prime}}\left\langle\hat{h}_{-\mathbf{k} n}^{\dagger} \hat{h}_{-\mathbf{k} m^{\prime}}\right\rangle-\mathbf{s}_{n m^{\prime}}^{*}\left\langle\hat{h}_{-\mathbf{k} m^{\prime}}^{\dagger} \hat{h}_{-\mathbf{k} n^{\prime}}\right\rangle\right] \\
& +\sum_{m^{\prime}} d_{m^{\prime} n}^{*}(t)\left\langle\hat{h}_{-\mathbf{k} n^{\prime}} \hat{e}_{\mathbf{k} m^{\prime}}\right\rangle-\sum_{m^{\prime}} d_{m^{\prime} n^{\prime}}(t)\left\langle\hat{h}_{-\mathbf{k} n} \hat{e}_{\mathbf{k} m^{\prime}}\right\rangle^{*} \\
& +\left.i \partial_{t}\left\langle\hat{h}_{-\mathbf{k} n}^{\dagger} \hat{h}_{-\mathbf{k} n^{\prime}}\right\rangle\right|_{\text {relax }} .
\end{aligned}
$$

The second line on the rhs of the above equation describes the change in the hole states due to the exchange interaction. The third line describes the excitation of hole coherences and populations by the optical pulse, via second-order Raman processes. Similarly, we obtain for the electron populations and coherences

$$
\begin{aligned}
& i \partial_{t}\left\langle\hat{e}_{\mathbf{k} n}^{\dagger} \hat{e}_{\mathbf{k} n^{\prime}}\right\rangle=\left(\varepsilon_{\mathbf{k} n^{\prime}}^{c}-\varepsilon_{\mathbf{k} n}^{c}\right)\left\langle\hat{e}_{\mathbf{k} n}^{\dagger} \hat{e}_{\mathbf{k} n^{\prime}}\right\rangle \\
& +\sum_{m^{\prime}} d_{n m^{\prime}}^{*}(t)\left\langle\hat{h}_{-\mathbf{k} m^{\prime}} \hat{e}_{\mathbf{k} n^{\prime}}\right\rangle-\sum_{m^{\prime}} d_{n^{\prime} m^{\prime}}(t)\left\langle\hat{h}_{-\mathbf{k} m^{\prime}} \hat{e}_{\mathbf{k} n}\right\rangle^{*} \\
& +\left.i \partial_{t}\left\langle\hat{e}_{\mathbf{k} n}^{\dagger} \hat{e}_{\mathbf{k} n^{\prime}}\right\rangle\right|_{\text {relax }} .
\end{aligned}
$$

The total carrier populations with given momentum $\mathbf{k}$,

$$
N_{\mathbf{k}}^{e}=\sum_{n}\left\langle\hat{e}_{\mathbf{k} n}^{\dagger} \hat{e}_{\mathbf{k} n}\right\rangle, N_{\mathbf{k}}^{h}=\sum_{n}\left\langle\hat{h}_{-\mathbf{k} n}^{\dagger} \hat{h}_{-\mathbf{k} n}\right\rangle,
$$

are not affected by the magnetic exchange interaction, which within the mean field approximation only changes 
the spin. They satisfy equations of motion similar to the Bloch equations:

$$
\partial_{t} N_{-\mathbf{k}}^{h}=2 \operatorname{Im} \sum_{n n^{\prime}} d_{n^{\prime} n}^{*}(t)\left\langle\hat{h}_{-\mathbf{k} n} \hat{e}_{\mathbf{k} n^{\prime}}\right\rangle-\left(N_{-\mathbf{k}}^{h}-f_{-\mathbf{k}}^{h}\right) / T_{1}^{h}
$$

and

$$
\partial_{t} N_{\mathbf{k}}^{e}=2 I m \sum_{n n^{\prime}} d_{n^{\prime} n}^{*}(t)\left\langle\hat{h}_{-\mathbf{k} n} \hat{e}_{\mathbf{k} n^{\prime}}\right\rangle-N_{\mathbf{k}}^{e} / T_{1}^{e},
$$

where $T_{1}^{h}$ and $T_{1}^{e}$ are the spin-independent population relaxation times, due to the carrier-carrier and carrierphonon scattering processes which thermalize the carrier system. The hole population relaxes to the thermal distribution $f_{\mathbf{k}}^{h}$.

Using Eq.(9) for the total hole spin, we obtain from Eq.(10) after some algebra that

$$
\partial_{t} \mathbf{s}_{\mathbf{k}}^{h}=\beta c \mathbf{S} \times \mathbf{s}_{\mathbf{k}}^{h}+\operatorname{Im} \mathbf{h}_{\mathbf{k}}(t)+i\left\langle\left[K_{h}, \mathbf{s}_{\mathbf{k}}^{h}\right]\right\rangle+\left.\partial_{t} \mathbf{s}_{\mathbf{k}}^{h}\right|_{\text {relax }} .
$$

The first term on the rhs describes the precession of the hole spin around the mean field created by the Mn spin. The second term describes the photoexcitation of hole spin via second-order Raman processes, which are determined by the transition matrix elements (selection rules) and the interband optical polarizations:

$$
\mathbf{h}_{\mathbf{k}}(t)=2 \sum_{n n^{\prime} m^{\prime}} d_{n m^{\prime}}^{*}(t) \mathbf{s}_{m^{\prime} n^{\prime}}\left\langle\hat{h}_{-\mathbf{k} n^{\prime}} \hat{e}_{\mathbf{k} n}\right\rangle .
$$

The third term on the rhs of Eq.(15) arises when the valence band states are not eigenstates of the hole spin: $\left[\mathbf{s}_{\mathbf{k}}^{h}, K_{h}\right] \neq 0$. In this case, the hole spin dynamics depends on the valence band energies and is determined by the individual coherences, Eq.(10), between the different valence bands. In III-Mn-V semiconductors, the hole spin is not conserved due to the strong spin-orbit interaction. Recalling Eq.(8), we conclude that the mixing of the hole spin states and the valence bandstructure modifies the effective magnetic field experienced by the Mn spin as described by the third term on the rhs of Eq.(15).

The photoexcitation of carrier spin, which triggers the magnetization dynamics, is governed by the interband optical polarizations

$$
P_{\mathbf{k} n n^{\prime}}=\left\langle\hat{h}_{-\mathbf{k} n} \hat{e}_{\mathbf{k} n^{\prime}}\right\rangle,
$$

determined by the equations of motion

$$
\begin{aligned}
& i \partial_{t} P_{\mathbf{k} n n^{\prime}}=\left(\varepsilon_{\mathbf{k} n^{\prime}}^{c}+\varepsilon_{\mathbf{k} n}^{v}-\omega_{p}-i / T_{2}\right) P_{\mathbf{k} n n^{\prime}} \\
& +\beta c \mathbf{S} \cdot \sum_{m} \mathbf{s}_{n m} P_{\mathbf{k} m n^{\prime}} \\
& -d_{n^{\prime} n}(t)\left[1-\left\langle\hat{h}_{-\mathbf{k} n}^{\dagger} \hat{h}_{-\mathbf{k} n}\right\rangle-\left\langle\hat{e}_{\mathbf{k} n^{\prime}}^{\dagger} \hat{e}_{\mathbf{k} n^{\prime}}\right\rangle\right] \\
& +\sum_{m \neq n} d_{n^{\prime} m}\left\langle\hat{h}_{-\mathbf{k} m}^{\dagger} \hat{h}_{-\mathbf{k} n}\right\rangle+\sum_{m \neq n^{\prime}} d_{m n}\left\langle\hat{e}_{\mathbf{k} m}^{\dagger} \hat{e}_{\mathbf{k} n^{\prime}}\right\rangle \\
& +\left.i \partial_{t} P_{\mathbf{k} n n^{\prime}}\right|_{\text {relax }} .
\end{aligned}
$$

In the above equation, $\omega_{p}$ is the pump optical pulse central frequency and $T_{2}$ describes the spin-independent contribution to the polarization dephasing. $T_{2}$ can be quite short due to the disorder, which relaxes the momentum conservation by introducing a one-body potential. For weak disorder, this effect can be treated by introducing a dephasing time comparable to the momentum scattering time. The second line on the rhs of the above equation is due to the change of the hole states induced by the effective magnetic field $\beta c \mathbf{S}(t)$. The third line describes the Pauli-blocking nonlinearity (Phase Space Filling) ${ }^{40}$, while the fourth line describes the contribution of carrier coherences between the different bands. Finally, the last line describes the spin-dependent polarization dephasing due to the hole spin-flip interactions, discussed in the next section. We note from the above equations that the interband polarizations, valence band coherences, and hole states depend on the effective magnetic field proportional to $\mathbf{S}(t)$. This spin can deviate significantly from its ground state configuration in the case of strong photoexcitation, which in turn changes the hole states as compared to the ground state. Such light-induced deviations from equilibrium increase with photoexcitation intensity and are described non-perturbatively by solving numerically the above system of coupled equations without expanding in terms of the optical field.

\section{HOLE SPIN AND POLARIZATION DEPHASING}

\section{A. Lindblad formalism}

The equations derived in the previous section do not include dephasing and relaxation contributions. Within the semiconductor Bloch Equations $\underline{40}$ such effects are treated phenomenologically by introducing effective dephasing and relaxation times to the polarization and population equations of motion. Here we must also consider the dynamics of the carrier and Mn spins, which within the mean field approximation precess around each other while conserving their magnitudes. In the III-Mn-V system, the hole spin relaxation is strong, mainly due to the spin-orbit interaction. For example, the combination of spin-orbit coupling in the valence band and disorderinduced scattering between momentum states, leads to hole spin dephasing and relaxation times of the order of 10 's of fs $\underline{48,49}$ Such times are comparable to the hole spin precession period around the Mn spin and cannot be neglected.

Carrier spin relaxation is often described within the spin Bloch equations $\underline{49,50}$ However, hole spin relaxation also leads to interband polarization dephasing, and both effects must be treated on equal footing for our purposes here. For this we use the Lindblad semigroup description of dissipative quantum dynamics $\underline{42}$ Under the general assumptions of linear coupling between bath and system operators and Markovian/relaxation time ap- 
proximation, as well as density matrix positivity and semigroup-type time evolution, the relaxation contribution to the density matrix equation of motion can be expressed in the form ${ }^{42}$

$$
\begin{aligned}
\left.\partial_{t} \rho\right|_{\text {relax }}= & \Gamma_{\perp} \sum_{\mathbf{k}^{\prime} m^{\prime}}\left[2\left\langle L_{\mathbf{k}^{\prime} m^{\prime}}^{\dagger} \rho L_{\mathbf{k}^{\prime} m^{\prime}}\right\rangle\right. \\
& \left.-\left\langle L_{\mathbf{k}^{\prime} m^{\prime}}^{\dagger} L_{\mathbf{k}^{\prime} m^{\prime}} \rho\right\rangle-\left\langle\rho L_{\mathbf{k}^{\prime} m^{\prime}}^{\dagger} L_{\mathbf{k}^{\prime} m^{\prime}}\right\rangle\right]
\end{aligned}
$$

where $\Gamma_{\perp}$ is the spin dephasing rate. The Lindblad operators $L_{\mathbf{k} m}$ must be chosen to describe the relaxation processes at hand. In our case, the hole spin must relax towards the direction antiparallel to the Mn-spin $\mathbf{S}(t)$. As we show below, this can be achieved by choosing

$$
L_{\mathbf{k m}}=\hat{h}_{-\mathbf{k} m \Downarrow}^{\dagger} \hat{h}_{-\mathbf{k} m \Uparrow},
$$

where $\hat{h}_{\mathbf{k m} \Uparrow}^{\dagger}\left(\hat{h}_{\mathbf{k m} \Downarrow}^{\dagger}\right)$ creates a hole with spin parallel (anti-parallel) to the Mn spin $\mathbf{S}(t)$ and the index $m$ labels the different basis states.

\section{B. Hole spin dephasing and relaxation}

First we derive the hole spin dephasing and relaxation described by the Lindblad operator Eq.(20). Noting that the hole spin relaxes to a direction antiparallel to the $\mathrm{Mn}$ spin, it is useful to introduce its components parallel and perpendicular to the unit vector $\hat{\mathbf{S}}$ :

$$
\mathbf{s}_{\mathbf{k} m}^{h}=\mathbf{s}_{\mathbf{k} m \perp}^{h}+\hat{\mathbf{S}} s_{\mathbf{k} m \|}^{h},
$$

where $s_{\mathbf{k} m \|}^{h}=\hat{\mathbf{S}} \cdot \mathbf{s}_{\mathbf{k} m}^{h}$ and

$$
\mathbf{s}_{\mathbf{k} m \perp}^{h}=\hat{\mathbf{S}} \times\left(\mathbf{s}_{\mathbf{k} m}^{h} \times \hat{\mathbf{S}}\right) .
$$

As derived in Appendix A, the Lindblad operator Eq. (20) gives the following spin-Bloch equations:

$$
\begin{array}{r}
\left.\partial_{t} \mathbf{s}_{\mathbf{k} m \perp}^{h}\right|_{\text {relax }}=-\Gamma_{\perp} \mathbf{s}_{\mathbf{k} m \perp}^{h}, \\
\left.\partial_{t} \mathbf{s}_{\mathbf{k} m \|}^{h}\right|_{\text {relax }}=-\Gamma_{\|}\left(\mathbf{s}_{\mathbf{k} m \|}^{h}+m_{\mathbf{k} m}^{h}\right),
\end{array}
$$

where $\Gamma_{\|}=2 \Gamma_{\perp}$ is the spin relaxation rate and

$$
m_{\mathbf{k} m}^{h}=N_{\mathbf{k} m}^{h} / 2-\left(N_{\mathbf{k} m}^{h} / 2\right)^{2}+\left(\mathbf{s}_{\mathbf{k} m}^{h}\right)^{2}
$$

is the quasi-equilibrium hole spin value. $m^{h}$ corresponds to the maximum spin, $s_{\max }$, of $N^{h}$ holes, given by the relation $s_{\max }-s_{\max }^{2}=N^{h} / 2-\left(N^{h} / 2\right)^{2}$.

\section{Spin-dependent Polarization Dephasing}

The hole spin dephasing also dephases the interband optical polarizations. To describe this, it is useful to use the basis of hole spin eigenstates discussed above and define the interband electron-hole amplitudes

$$
P_{\mathbf{k} m n \sigma}=\left\langle\hat{h}_{-\mathbf{k} m \sigma} \hat{e}_{\mathbf{k} n}\right\rangle,
$$

where $\sigma=\uparrow, \downarrow$. Using Eqs. (19) and (20), we derive in Appendix (B) the following expression for the spindependent polarization dephasing:

$$
\begin{aligned}
& \left.\partial_{t} P_{\mathbf{k} m n \uparrow}\right|_{\text {relax }}=-\Gamma_{\perp} \times \\
& \left\{P_{\mathbf{k} m n \uparrow}\left[\frac{1}{2}+s_{\mathbf{k} m \|}^{h}+\frac{\hat{\mathbf{S}}_{z}}{2}\left(1-N_{\mathbf{k} m}^{h}\right)+i\left(\hat{\mathbf{S}} \times \mathbf{s}_{\mathbf{k} m}^{h}\right)_{z}\right]\right. \\
& \left.+P_{\mathbf{k} m n \downarrow}\left[\frac{\hat{\mathbf{S}}_{-}}{2}\left(1-N_{\mathbf{k} m}^{h}\right)+i\left(\hat{\mathbf{S}} \times \mathbf{s}_{\mathbf{k} m}^{h}\right)_{-}\right]\right\}, \\
& \left.\partial_{t} P_{\mathbf{k} m n \downarrow}\right|_{\text {relax }}=-\Gamma_{\perp} \times \\
& \left\{P_{\mathbf{k} m n \downarrow}\left[\frac{1}{2}+s_{\mathbf{k} m \|}^{h}-\frac{\hat{\mathbf{S}}_{z}}{2}\left(1-N_{\mathbf{k} m}^{h}\right)-i\left(\hat{\mathbf{S}} \times \mathbf{s}_{\mathbf{k} m}^{h}\right)_{z}\right]\right. \\
& \left.+P_{\mathbf{k} m n \uparrow}\left[\frac{\hat{\mathbf{S}}_{+}}{2}\left(1-N_{\mathbf{k} m}^{h}\right)+i\left(\hat{\mathbf{S}} \times \mathbf{s}_{\mathbf{k} m}^{h}\right)_{+}\right]\right\},
\end{aligned}
$$

where, for any vector $\mathbf{A}, A_{ \pm}=A_{x} \pm i A_{y}$. The above dephasing contribution depends nonlinearly on the photoexcitation, via the photoexcited hole contribution to the population $N^{h}$ and spin $\mathbf{s}^{h}$ and the light-induced changes in the Mn spin S. These nonlinearities correspond to excitation-induced dephasing induced by hole spin-flip correlations.

\section{SINGLE BAND APPROXIMATION}

The general theory derived in the previous sections may be used to treat the bandstructure relevant to the particular ferromagnetic material of interest. Below we discuss the general features of the dynamics, their physical origin, and their sensitivity to different parameters by extracting from the general theory a simplified oneband model that captures the essential physics common in all materials. We therefore neglect bandstructure particularities, such as the nature of the high energy states far from the Brillouin zone center, excited by the $\sim 3.1 \mathrm{eV}$ pump of Ref 35, or impurity bands. We assume that the magnetization dynamics is mainly triggered by optical transitions between a single valence and conduction band of spin- $\uparrow$ and spin- $\downarrow$ states, whose mixing is described phenomenologically with the Linbdlad approach. In the basis of carrier spin eigenstates and assuming a single band, Eq.(3) reduces to

$$
H_{L}=-d_{+} \sum_{\mathbf{k}} \hat{e}_{\mathbf{k} \downarrow}^{\dagger} \hat{h}_{-\mathbf{k} \uparrow}^{\dagger}-d_{-} \sum_{\mathbf{k}} \hat{e}_{\mathbf{k} \uparrow}^{\dagger} \hat{h}_{-\mathbf{k} \downarrow}^{\dagger}+\text { h.c. }
$$

where $d_{ \pm}(t)=\mu_{ \pm} \mathcal{E}(t)$. The Rabi energy $d_{+}$describes the coupling of the right-circularly polarized component of the optical field, while $d_{-}$describes the coupling of the 
left-circularly polarized component. The corresponding interband transition matrix elements $\mu_{ \pm}$depend on the bandstructure and the admixture of spin- $-\uparrow$ and spin $-\downarrow$ in the band states that mostly contribute to Eq.(3) for the energies of interest. Denoting

$$
P_{\mathbf{k} \sigma}^{+}=\left\langle\hat{h}_{-\mathbf{k} \sigma} \hat{e}_{\mathbf{k} \downarrow}\right\rangle, P_{\mathbf{k} \sigma}^{-}=\left\langle\hat{h}_{-\mathbf{k} \sigma} \hat{e}_{\mathbf{k} \uparrow}\right\rangle .
$$

and using Eqs.(27) and (28) and the results of Section [III, we obtain the following coupled equations of motion for the interband optical polarizations:

$$
\begin{aligned}
& i \partial_{t} P_{\mathbf{k} \uparrow}^{+}-\left(\Omega_{\mathbf{k}}+\boldsymbol{\Delta}_{\mathbf{k} z}\right) P_{\mathbf{k} \uparrow}^{+}-\boldsymbol{\Delta}_{\mathbf{k}-} P_{\mathbf{k} \downarrow}^{+} \\
& =-d_{+}(t)\left(1-n_{\mathbf{k} \downarrow}^{e}-n_{\mathbf{k} \uparrow}^{h}\right), \\
& i \partial_{t} P_{\mathbf{k} \downarrow}^{+}-\left(\Omega_{\mathbf{k}}-\boldsymbol{\Delta}_{\mathbf{k} z}\right) P_{\mathbf{k} \downarrow}^{+}-\boldsymbol{\Delta}_{\mathbf{k}+} P_{\mathbf{k} \uparrow}^{+} \\
& =d_{+}(t) \mathbf{s}_{\mathbf{k}+}^{h}+d_{-}(t) \mathbf{s}_{\mathbf{k}+}^{e}, \\
& i \partial_{t} P_{\mathbf{k} \downarrow}^{-}-\left(\Omega_{\mathbf{k}}-\boldsymbol{\Delta}_{\mathbf{k} z}\right) P_{\mathbf{k} \downarrow}^{-}-\boldsymbol{\Delta}_{\mathbf{k}+} P_{\mathbf{k} \uparrow}^{-} \\
& =-d_{-}(t)\left(1-n_{\mathbf{k} \uparrow}^{e}-n_{\mathbf{k} \downarrow}^{h}\right), \\
& i \partial_{t} P_{\mathbf{k} \uparrow}^{-}-\left(\Omega_{\mathbf{k}}+\boldsymbol{\Delta}_{\mathbf{k} z}\right) P_{\mathbf{k} \uparrow}^{-}-\boldsymbol{\Delta}_{\mathbf{k}-} P_{\mathbf{k} \downarrow}^{-} \\
& =d_{-}(t) \mathbf{s}_{\mathbf{k}-}^{h}+d_{+}(t) \mathbf{s}_{\mathbf{k}-}^{e},
\end{aligned}
$$

where

$$
\Omega_{\mathbf{k}}=\varepsilon_{\mathbf{k}}^{v}+\varepsilon_{\mathbf{k}}^{c}-\omega_{p}-i\left[\frac{1}{T_{2}}+\Gamma_{\perp}\left(\frac{1}{2}+s_{\mathbf{k} \|}^{h}\right)\right]
$$

gives the Mn spin-independent and

$$
\boldsymbol{\Delta}_{\mathbf{k}}=\frac{\hat{\mathbf{S}}}{2}\left[\beta c S-i \Gamma_{\perp}\left(1-N_{\mathbf{k}}^{h}\right)\right]+\Gamma_{\perp} \hat{\mathbf{S}} \times \mathbf{s}_{\mathbf{k}}^{h}
$$

the Mn spin-dependent contribution to the e- $\mathrm{h}$ pair energy and dephasing. $\boldsymbol{\Delta}_{\mathbf{k}}$ also determines the coupling between the interband polarizations, due to the mixing of the hole spins by the magnetic exchange interaction and the dephasing. The above $\mathrm{e}-\mathrm{h}$ pair energies depend on the light-induced deviations of the Mn and hole spins from their ground state configurations and on the photoexcited hole populations, which give nonlinear contributions to the optical polarization. The rhs of Eqs.(31) and (33) describes the Pauli-blocking nonlinearities (Phase Space Filling) ${ }^{40}$, while the rhs of Eqs.(32) and (34) describes the contribution of carrier spin coherences, ground state or photoexcited. The above polarization equations of motion treat the effects of the mixing of the spin- $\uparrow$ and spin- $\downarrow$ states by spin-orbit or other spin-flip interactions by using Eqs.(27) and (28).

Finally we turn to the equations of motion for the carrier spins. The hole spin dynamics is described by Eq.(15). Within the one-band approximation, the mixing of the spin- $\uparrow$ and spin- $-\downarrow$ hole states leads to the spin dephasing derived in Appendix A The second term on the rhs of Eq. (15) describes the photoexcitation of hole spin as determined by the interband polarizations and $\mathbf{h}_{\mathbf{k}}(t)$, Eq.(16). In the basis of hole spin eigestates con- sidered in this section,

$$
\begin{aligned}
& \mathbf{h}_{\mathbf{k} x}(t)=d_{+}^{*}(t) P_{\mathbf{k} \downarrow}^{+}(t)+d_{-}^{*}(t) P_{\mathbf{k} \uparrow}^{-}(t) \\
& \mathbf{h}_{\mathbf{k} y}(t)=-i\left[d_{+}^{*}(t) P_{\mathbf{k} \downarrow}^{+}(t)-d_{-}^{*}(t) P_{\mathbf{k} \uparrow}^{-}(t)\right], \\
& \mathbf{h}_{\mathbf{k} z}(t)=d_{+}^{*}(t) P_{\mathbf{k} \uparrow}^{+}(t)-d_{-}^{*}(t) P_{\mathbf{k} \downarrow}^{-}(t) .
\end{aligned}
$$

The above equation describes a second-order nonlinear optical process where the excitation of a spin- $\downarrow$ holespin- $\downarrow$ electron pair is followed by the de-excitation of a spin- $\uparrow$ hole-spin- $\downarrow$ electron pair. This second-order (Raman) process is induced by the right-circularly polarized component of the optical field, while the leftcircularly polarized component induces an analogous process involving the electron spin- $\uparrow$ states. The above process requires a nonzero polarization $P_{\mathbf{k} \downarrow}^{+}$. This is possible on the one hand due to the coupling of the spin- $\uparrow$ and spin- $\downarrow$ hole states (and hence $P_{\mathbf{k} \downarrow}^{+}$with $P_{\mathbf{k} \uparrow}^{+}$, third term on the lhs of Eq.(32) described by $\boldsymbol{\Delta}_{\mathbf{k}}$, due to the magnetic exchange interaction and the hole spin dephasing processes (e.g. spin-orbit), and on the other hand due to the presence of spin- $\uparrow$-spin- $\downarrow$ hole spin coherence in the ground state. Finally, Eq.(37) describes the photoexcitation of spin-polarized hole populations and a hole spin $\mathrm{z}$-component via the excitation and subsequent de-excitation of a spin- $-\uparrow$ hole and spin- $-\downarrow$ electron pair (right-circularly polarized component) or a spin- $\downarrow$ hole and spin- $\uparrow$ electron pair (left-circularly polarized component). The above coherent nonlinear effects occur during the optical pulse and do not require the absorption of light. They can also be induced by photoexciting the system below resonance, in the transparency regime, provided that the photoexcitation intensity is sufficiently high to achieve an observable effect. Such below-resonance photoexcitation is advantageous since undesirable effects such as transient heating can be suppressed and thus the speed of a possible device can be maximized. The equation of motion for the electron spin has a form similar to Eq.(15):

$$
\left(\partial_{t}+\Gamma_{e}^{s}\right) \mathbf{s}_{\mathbf{k}}^{e}=\operatorname{Im} \mathbf{h}_{\mathbf{k}}^{e}(t)
$$

where $\Gamma_{e}^{s}$ is the electron spin dephasing rate and $\mathbf{h}_{\mathbf{k}}^{e}$ describes the photoexcitation of conduction electron spin:

$$
\begin{aligned}
& \mathbf{h}_{\mathbf{k} x}^{e}(t)=d_{-}^{*}(t) P_{\mathbf{k} \downarrow}^{+}(t)+d_{+}^{*}(t) P_{\mathbf{k} \uparrow}^{-}(t), \\
& \mathbf{h}_{\mathbf{k} y}^{e}(t)=i\left[d_{+}^{*}(t) P_{\mathbf{k} \uparrow}^{-}(t)-d_{-}^{*}(t) P_{\mathbf{k} \downarrow}^{+}(t)\right], \\
& \mathbf{h}_{\mathbf{k} z}^{e}(t)=-\left[d_{+}^{*}(t) P_{\mathbf{k} \uparrow}^{+}(t)-d_{-}^{*}(t) P_{\mathbf{k} \downarrow}^{-}(t)\right] .
\end{aligned}
$$

In the next section we calculate the Mn spin dynamics by solving the above system of coupled equations nonperturbatively, which allows us to treat large deviations of the Mn spin from its ground state configuration induced by strong photoexcitation.

\section{NUMERICAL RESULTS}

Within the mean field approximation, light-induced Mn spin dynamics is triggered initially by the photoex- 
(a)

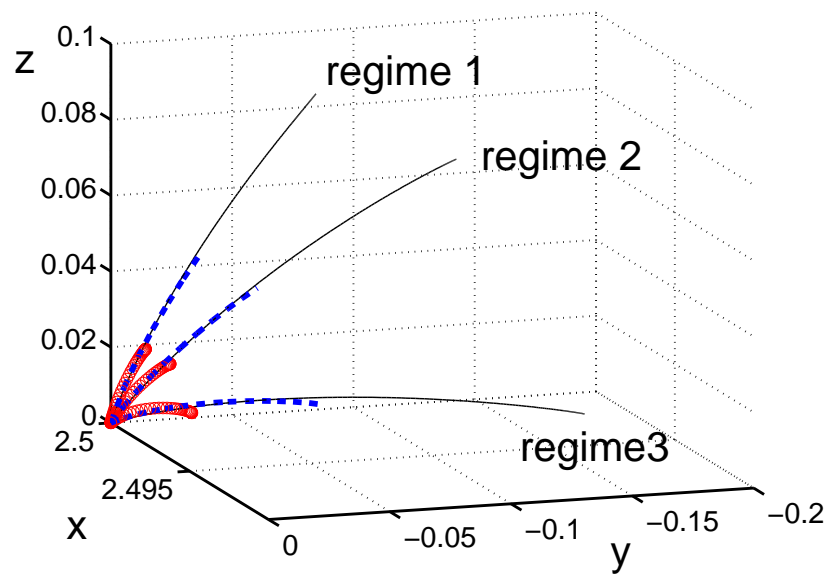

(b)

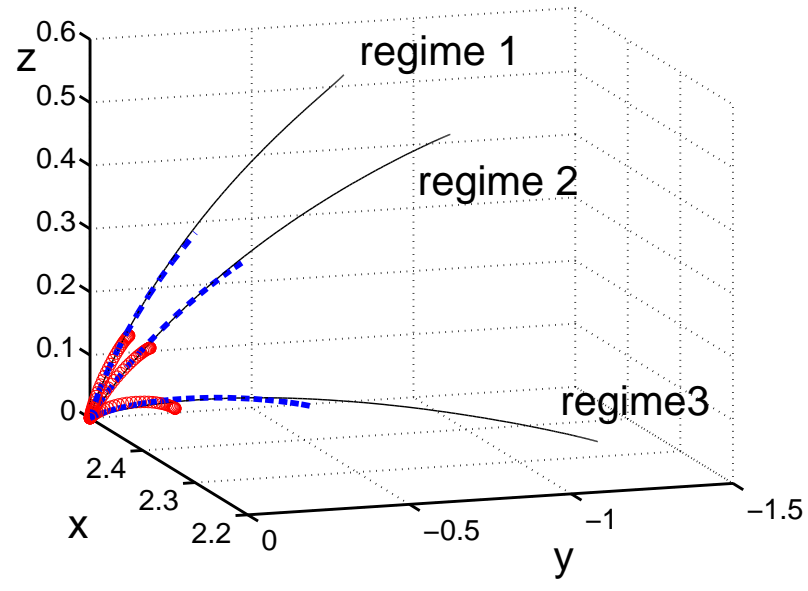

FIG. 1: (Color online) Mn spin trajectories without magnetic anisotropy in the three regimes of spin and polarization dephasing discussed in the text for three pulse durations $\tau_{p}$ and two different Rabi energies: (a) $d_{+}(0)=20 \mathrm{meV}$, (b) $d_{+}(0)=60 \mathrm{meV}$. Thick circled line: $\tau_{p}=100 \mathrm{fs}$, Dashed line: $\tau_{p}=250 \mathrm{fs}$, Thin solid line: $\tau_{p}=500 \mathrm{fs}$.

citation of a hole spin component perpendicular to the ground state Mn spin. In the simple one-band approximation, the magnitude of such a spin component is determined by the ratio $d_{+} / d_{-}$between the Rabi energies that describe the coupling of the right- and left-circularly polarized components of the optical field. This ratio is determined by the selection rules and the nature of the bands that contribute to the magnetization dynamics. Within our simplified one-band model, $d_{+} / d_{-}$also reflects the magnetic anisotropy of the system due to the bandstructure. In the absence of a complete theoretical understanding of such bandstructure effects, especially for the high energy $(\sim 3.1 \mathrm{eV})$ transitions observed experimentally to trigger ultrafast magnetization dynamics in $\mathrm{Ga}(\mathrm{Mn}) \mathrm{As}, \stackrel{35}{5}$ we consider here the extreme case of $d_{-}=0$, which corresponds to right-circularly polarized light. For all the calculations presented in this pa- per, we consider a magnetic exchange energy $\beta c S=125$ meV comparable to the Fermi energy $E_{F}=100 \mathrm{meV}$, fraction of initial holes 0.33 of the Mn impurities, a hole mass $m_{h}=7.15 m_{e}$, and a carrier thermalization time $T_{1}=165 \mathrm{fs}$. Our results are not very sensitive to the above parameters, with the exception of the exchange interaction $\beta$ that changes the magnitude of the effect. To make the connection between the calculated quantities and the experiment, we note that ultrafast magnetooptical pump-probe spectroscopy can be used to deduce the time evolution of the $\mathrm{z}$-component of the magnetization, $\sim S_{z}(t) \stackrel{7.35}{.35}$

We start with our results in the absence of magnetic anisotropy. Fig [1]shows the light-induced time evolution of the Mn spin and its dependence on the hole spin and polarization dephasing, the Rabi energy, and the optical pulse duration. By comparing three different dephasing regimes in Fig 1, one can see that the shape of the Mn spin trajectory depends sensitively on the hole spin and polarization dephasing. Regime 1 corresponds to very short dephasing times, $1 / \Gamma_{\perp}=21 \mathrm{fs}$ and $T_{2}=10.5 \mathrm{fs}$, which

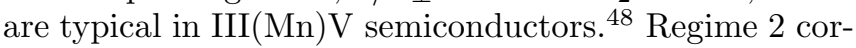
responds to $T_{2}=330 \mathrm{fs}$ with very short hole spin dephasing $1 / \Gamma_{\perp}=21 \mathrm{fs}$. Finally, Regime 3 neglects the spin dephasing altogether, $\Gamma_{\perp}=0, T_{2}=330 \mathrm{fs}$, which assumes that the hole spin is a good quantum number. As can be seen in Fig 1, the dephasing changes qualitatively the shape of the Mn spin trajectory, from the precession within the $\mathrm{x}-$ $y$ plane expected in the absence of magnetic anisotropy to a complex magnetization tilt out of the $\mathrm{x}-\mathrm{y}$ plane, in the direction of pulse propagation. The magnitude of this out-of-plane tilt and the Mn spin z-component are enhanced by increasing $\Gamma_{\perp}$ or the pulse duration $\tau_{p}$ and by the short $T_{2}$. Furthermore, the Mn spin component within the $\mathrm{x}-\mathrm{y}$ plane rotates in a clockwise direction, opposite to the counter-clockwise direction expected for an effective magnetic field along the $+z$ axis of pulse propagation. For right-circular-polarization, the latter is the expected direction of the photoexcited hole spin in the absence of magnetic exchange interaction or spin dephasing. However, our numerical results show that the photoexcited hole spin $\mathrm{z}^{-}$-component is in fact negative, due to the magnetic exchange interaction and the mixing of the hole spin states. The dependence of the trajectories in Fig 1 on the pulse duration shows that the light-induced $\mathrm{Mn}$ spin dynamics terminates soon after the end the photoexcitation, as expected in the absence of magnetic anisotropy. Furthermore, the magnitude of the Mn spin tilt increases with photoexcitation intensity. The spin dynamics of Fig 1describes the fundamental response of the spin system to the optical excitation within the mean field approximation.

The Mn spin response is seen more clearly in Fig, 2 , which shows the development with time of the $\mathrm{y}^{-}$and $\mathrm{z}^{-}$ components of $\mathbf{S}$. Both of these components vanish in the ground state. They develop on a timescale determined by the pulse duration and have comparable magnitudes for sufficiently large $\Gamma_{\perp}$. The importance of the hole spin 

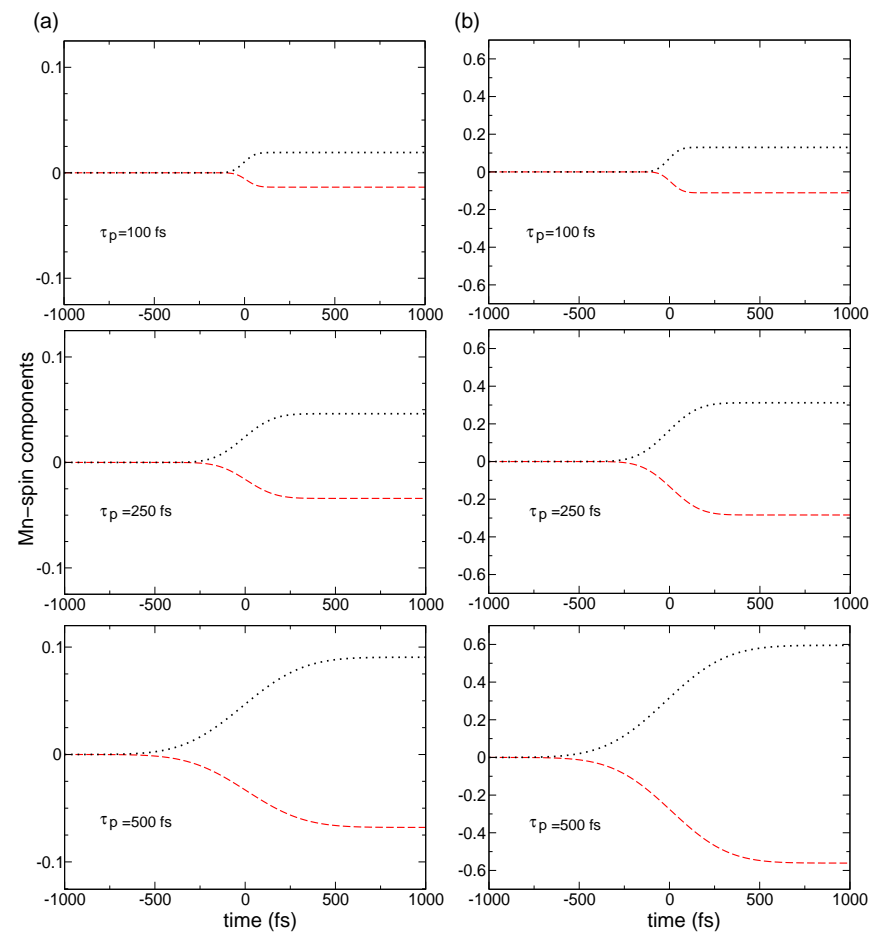

FIG. 2: (Color online) Mn spin components without magnetic anisotropy for pulse durations and Rabi energies as in Fig[1 $1 / \Gamma_{\perp}=21 \mathrm{fs}, T_{2}=10.5 \mathrm{fs}$. Dashed line: $\mathbf{S}_{y}(t)$. Dotted line: $\mathbf{S}_{z}(t)$

dephasing can be seen by comparing Fig 2, obtained for very short spin and polarization dephasing times, with Fig 3, obtained for $\Gamma_{\perp}=0$. The most striking difference is the very small magnitude of the out-of-plane component $S_{z}(t)$ when the hole spin is conserved $\left(\Gamma_{\perp}=0\right)$. The hole spin follows the overall $\mathrm{Mn}$ spin and remains more or less antiparallel to $\mathbf{S}(t)$ at all times, with the exception of a small component perpendicular to $\mathbf{S}$ that triggers the spin dynamics.

We now turn to the effects of the magnetic anisotropy on the spin dynamics. As discussed above, the magnetic anisotropy of the thermal carriers leads to preferred Mn spin directions that minimize the total Fermi sea energy $E_{h}(\mathbf{S})$, Eq.(5). Its effects on the Mn spin dynamics can be treated phenomenologically by adding the magnetic field

$$
\mathbf{H}(t)=-\frac{\partial E_{h}(\hat{\mathbf{S}})}{\partial \hat{\mathbf{S}}}
$$

to Eq. (8), $: 50,51$ Such an anisotropy field causes a nonlinear rotation and relaxation of the Mn spin until it aligns with the easy axis direction that minimizes $E_{h}(\hat{\mathbf{S}})$, so that $\mathbf{H}=0$. We would like to distinguish between the above effect, due to the thermal carriers, and the anisotropy effects on the photoexcitation. The latter can be treated microscopically as discussed in Section III and determine the photoexcited hole spin (see e.g. Eq.(15)).

In $\mathrm{Ga}(\mathrm{Mn}) \mathrm{As}, K_{c}<0, K_{u}>0$, and $\left|K_{c}\right|>K_{u} \stackrel{46,47}{ } \mathrm{In}$

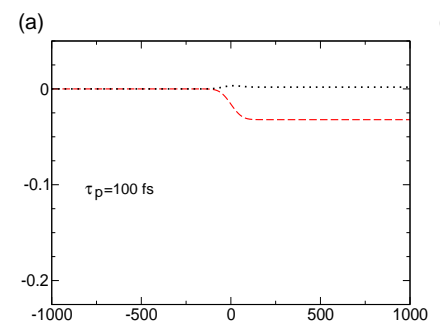

(b)
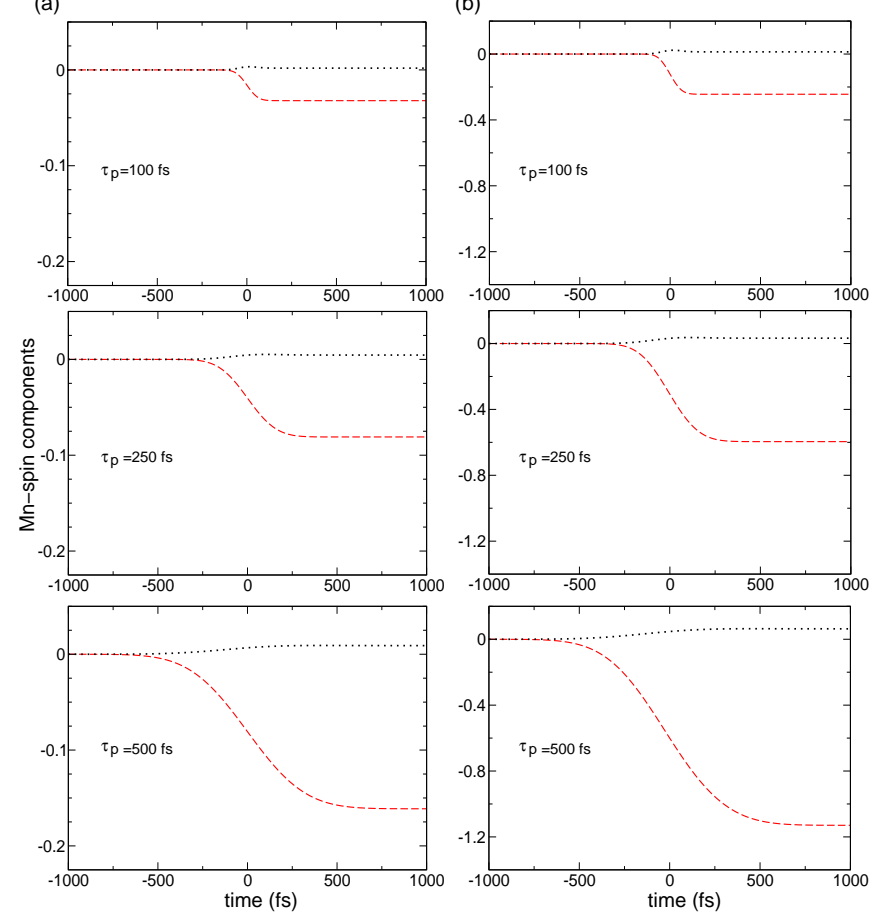

FIG. 3: (Color online) Time evolution of Mn spin components for the parameters of Fig 2 except for $\Gamma_{\perp}=0, T_{2}=330 \mathrm{fs}$.

this parameter regime, there are two degenerate ground states, which correspond to Mn spin pointing at angles $\phi$ from the $\mathrm{x}$-axis such that $\cos 2 \phi=K_{u} /\left|K_{c}\right|$. The system can be prepared so that, prior to the photoexcitation, the Mn spin points along either one of the above two easy axes. In the absence of magnetic anisotropy, both these initial conditions would result in the same $\mathrm{Mn}$ spin trajectory. Figs 4 (a) and 4 (b) compare the three Mn spin components as function of time, starting with $\mathrm{Mn}$ spin pointing along the two different ground state configurations. To make this comparison more meaningful, in both cases we chose the $\mathrm{x}$-axis of the coordinate system to coincide with the initial magnetization direction. Fig 4 clearly shows that, as the Rabi energy increases, the two initial conditions lead to different time evolution, which implies that the two magnetic ground states can be distinguished. As can be seen by comparing Fig 4 with Fig 5 the Mn spin dynamics depends on the hole spin dephasing, especially as the photoexcitation intensity increases.

The dependence of the Mn spin dynamics on the initial magnetic state becomes more clear in Figs 6 and 7 , which plot the Mn spin trajectories for the two initial conditions in the same coordinate system, whose $\mathrm{x}$-axis coincides with the initial magnetizations. Two different temporal regimes can be clearly distinguished. The first regime lasts for a time interval comparable to the pulse duration. Here the dynamics is governed by the optical pulse intensity, duration, and helicity, as well as by the ratio $\mu_{+} / \mu_{-}$and the mixing of the hole spin states 

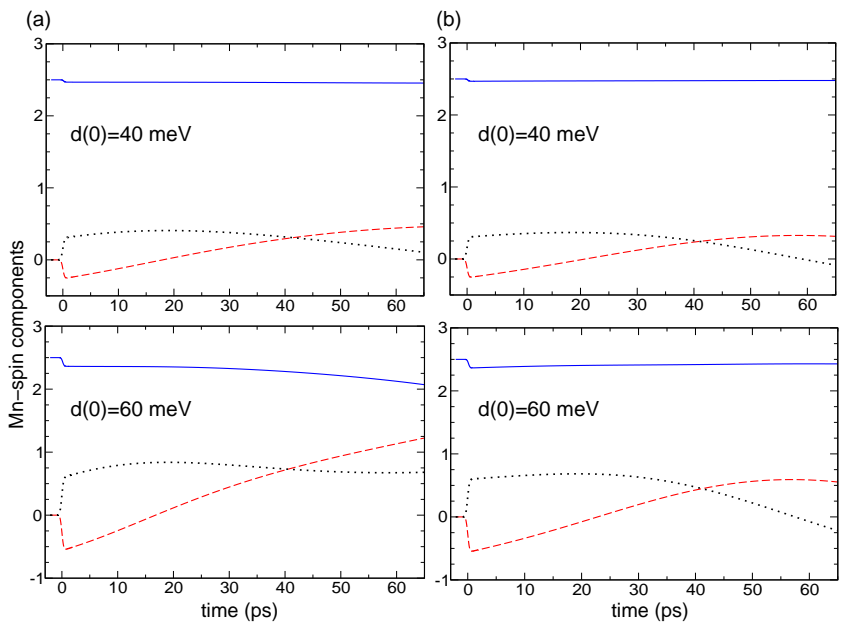

FIG. 4: (Color online) Magnetic anisotropy effects on the Mn spin components for strong dephasing $1 / \Gamma_{\perp}=21 \mathrm{fs}, T_{2}=10.5 \mathrm{fs}$, pulse duration $t_{p}=500 \mathrm{fs}$, and two Rabi energies. Solid line: $\mathbf{S}_{x}(t)$. Dashed line: $\mathbf{S}_{y}(t)$. Dotted line: $\mathbf{S}_{z}(t)$. (a) and (b) compare the time evolution for initial condition along the two easy axes, taken as the $\mathrm{x}$-axis. $K_{c}=-0.0175 \mathrm{meV}$, $K_{u z}=0.0252 \mathrm{meV}, K_{u}=0.010 \mathrm{meV}$.

that depend on the magnetic anisotropy. On the other hand, the magnetic anisotropy field Eq. (40), due to the thermal carriers, plays a very minor role in this initial temporal regime for sufficiently large Rabi energies that exceed the anisotropy constants. The trajectories for the different initial conditions coincide in this sub-picosecond regime, which gives an ultrafast magnetization tilt determined by the response to the hole spin photoexcited by the coherent spin Raman processes described by Eqs. (15) and (37). As can be seen by comparing Figs 6 and 7 the shape of the Mn spin trajectories is strongly influenced by the spin and polarization dephasing.

Figs [6 and [7 show a rather abrupt change in the $\mathrm{Mn}$ spin trajectory at times $t \sim \tau_{p}$. At later times, the temporal evolution is determined by the magnetic anisotropy field Eq.(40). The shape of the trajectory during many picoseconds depends critically on the magnetization tilt that develops during the femtosecond initial stage. If this tilt is sufficiently small, the Mn spin dynamics is described by linearized equations of motion, expanded around the equilibrium spin values that minimize the Fermi sea magnetic energy $E_{h}(\mathbf{S})$. This harmonic oscillation corresponds to zero-momentum magnon excitations, whose frequency is the same for the two easy axes. Since the magnitude of the magnetization tilt can be controlled by the optical pulse intensity and duration, we conclude that for sufficiently short pulses and sufficiently small Rabi energies, $d_{+} \tau_{p}<1$, the Mn spin trajectories are very similar for both initial conditions. They correspond to magnetization precession around the easy axis (magnons). However, with increasing photoexcitation intensity and duration, the deviation from the easy axis due to the sub-picosecond magnetization tilt increases, and

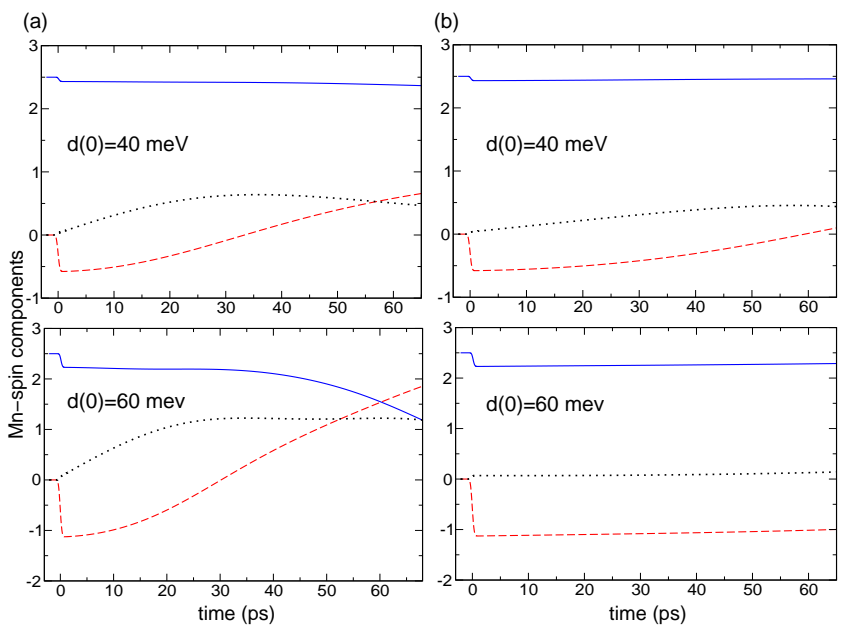

FIG. 5: (Color online) Time evolution of Mn spin components for the parameters of Fig 4 except for $\Gamma_{\perp}=0, T_{2}=330 \mathrm{fs}$.

eventually the Mn spin dynamics cannot be described by expanding $E_{h}(\mathbf{S})$ around its minimum. Such nonlinearities result in a complex trajectory, determined by the full nonlinear equations Eqs. (8) and (40), which can differ substantially from a simple magnon precession. As demsonstrated by Figs 6 and 7 , in this nonlinear dynamics regime, the Mn spin trajectories depend on the initial condition. One can then distinguish between the different magnetic states, which can provide the basis for an ultrafast magnetic memory read-out scheme with speed limited only by the optical pulse duration. Such a scheme would be based on controlling the ultrafast response of the spin system via the optical pulse intensity, duration, and helicity. Furthermore, the strong dependence of the trajectory shape on the spin dephasing implies that the valence bandstructure and mixing of the hole spin states by the spin orbit interaction plays an important role. In the next section we interpret our numerical results by deriving from the full theory an effective Landau-Gilbertlike equation for the Mn spin after expanding around the adiabatic limit.

\section{INTERPRETATION: LIGHT-INDUCED RELAXATION AND RE-ORIENTATION}

The numerical solution of the full mean field equations, discussed in the previous section, shows that the hole spin follows the Mn spin more or less adiabatically. This is due to the much faster hole spin precession and relaxation as compared to the time-dependent changes in $\mathbf{S}(t)$. To interpret the full numerical results, we therefore expand around the adiabatic limit. The derivation presented in Appendix Clthen gives a Landau-Gilbert-like ${ }^{43}$ equation:

$$
\begin{aligned}
\partial_{t} \hat{\mathbf{S}} & =\left(1-\gamma s_{\|}^{h}\right) \hat{\mathbf{S}} \times \mathbf{H}+\alpha s_{\|}^{h} \hat{\mathbf{S}} \times(\hat{\mathbf{S}} \times \mathbf{H}) \\
& -\alpha \hat{\mathbf{S}} \times \mathbf{h}(t)-\gamma \hat{\mathbf{S}} \times(\hat{\mathbf{S}} \times \mathbf{h}(t)),
\end{aligned}
$$


(a)

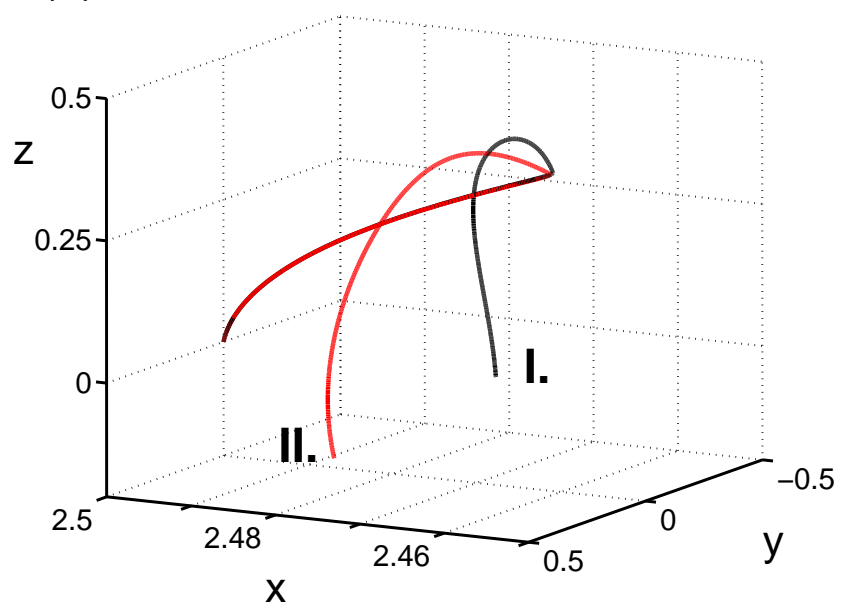

(b)

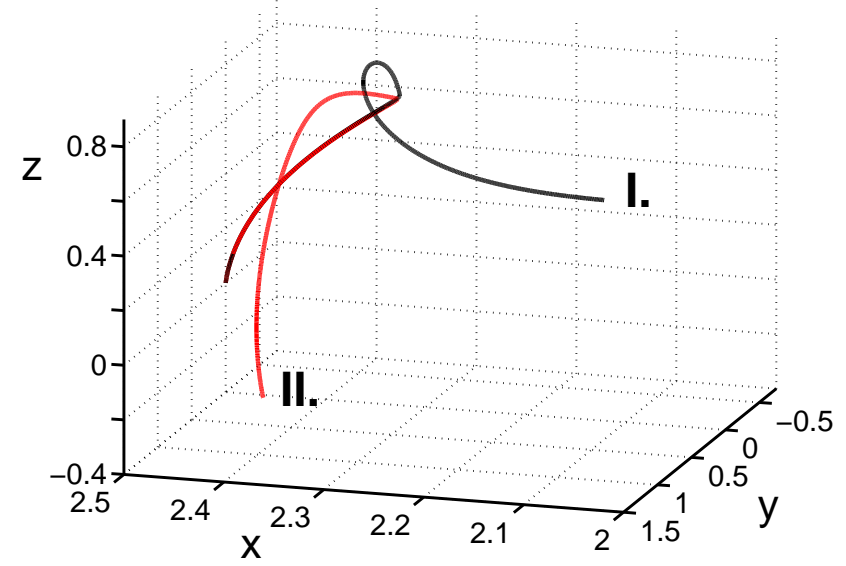

FIG. 6: (Color online) Mn spin trajectories with initial condition along the two different easy axes (I and II) for the parameters of Fig 4 (a): $d_{+}=40 \mathrm{meV},(\mathrm{b}): d_{+}=60 \mathrm{meV}$.

where the effective magnetic fields $\mathbf{h}$ (Eq.(37)),

$$
\mathbf{h}(t)=\frac{1}{V} \sum_{\mathbf{k}} \operatorname{Im} \mathbf{h}_{\mathbf{k}}(t)
$$

and $\mathbf{H}$, Eq. (40), are due to the optical excitation and magnetic anisotropy respectively. In the above equation,

$$
\gamma=\frac{\beta\left(\beta c S+\beta s_{\|}^{h}+H_{\|}\right)}{\left(\beta c S+\beta s_{\|}^{h}+H_{\|}\right)^{2}+\Gamma_{\perp}^{2}}
$$

where $H_{\|}=\mathbf{H} \cdot \hat{\mathbf{S}}$, while

$$
\alpha=\frac{\Gamma_{\perp} \beta}{\left(\beta c S+\beta s_{\|}^{h}+H_{\|}\right)^{2}+\Gamma_{\perp}^{2}} .
$$

The first two terms on the rhs of Eq.(41) correspond to the usual Landau-Gilbert description of the Mn spin dynamics induced by the magnetic anisotropy field $\mathbf{H}$. They

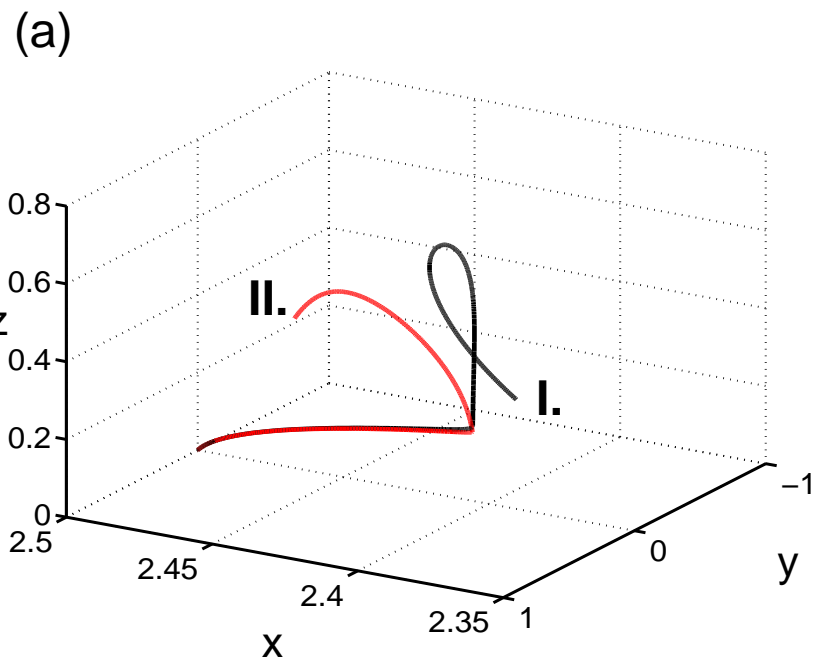

(b)

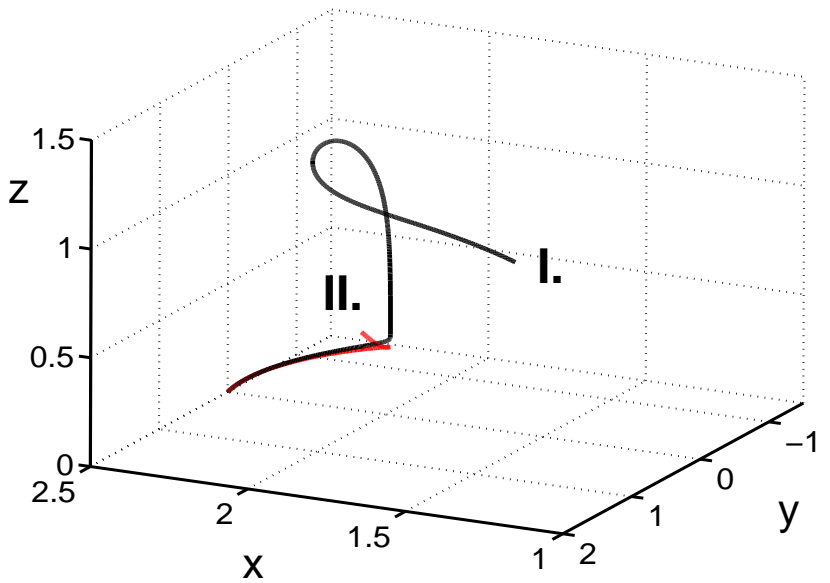

FIG. 7: (Color online) Mn spin trajectories for the same parameters as in Fig 6 except for $\Gamma_{\perp}=0, T_{2}=330 \mathrm{fs}$.

are similar to the results of Refs. $50,52,53$ and also include nonlinear corrections. The first term on the rhs describes the Mn spin rotation around the anisotropy field H. $\gamma$, Eq.(43), gives the renormalization of the gyromagnetic ratio by the magnetic exchange interaction. The second term describes the Gilbert relaxation of the $\mathrm{Mn}$ spin towards the direction of $\mathbf{H}$, with Gilbert damping coefficient $\propto \alpha$, Eq.(44). The precise values of $\gamma$ and $\alpha$ in the realistic system depend on the details of the valence bandstructure. The relaxation of the hole spin component parallel to $\mathbf{S}(t), s_{\|}^{h}$, depends on the two fields $\mathbf{h}(t)$ (nonlinear optical excitation) and $\mathbf{H}(t)$ (magnetic anisotropy) as described in Appendix D.

The nonlinear optical excitation gives rise to two additional contributions to the Mn spin equation of motion Eq.(41). These contributions describe the response of the magnetization to the effective magnetic field pulse $\mathbf{h}(t)$, which is generated by the optical excitation via second order processes. The third term on the rhs of Eq. (41) describes a $\mathrm{Mn}$ spin rotation around $\mathbf{h}(t)$, whose magni- 
(a)

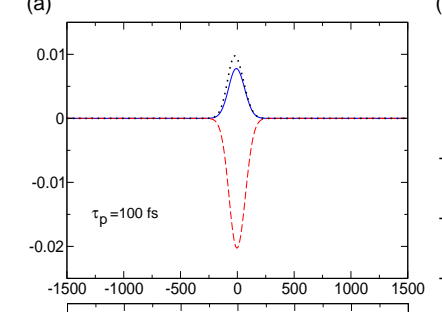

(b)
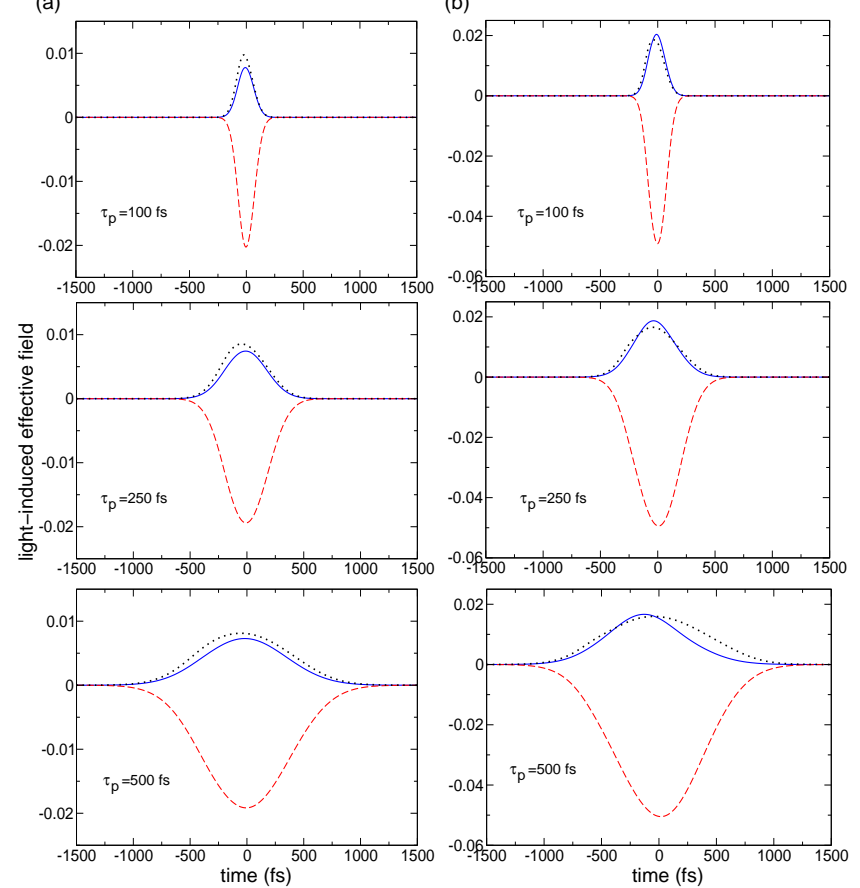

FIG. 8: (Color online) Light-induced effective magnetic field components for pulse durations and Rabi energies as in Fig 1 $1 / \Gamma_{\perp}=21 \mathrm{fs}, T_{2}=10.5 \mathrm{fs}$. Solid line: $\mathbf{h}_{x}(t)$, Dashed line: $\mathbf{h}_{y}(t)$, Dotted line: $\mathbf{h}_{z}(t)$.

tude is proportional to the Gilbert damping coefficient $\alpha$, Eq. (44), and therefore vanishes if $\Gamma_{\perp}=0$ (hole spin conserved). The last term describes a spin relaxation towards $\mathbf{h}(t)$, determined by the exchange interaction. The above light-induced rotation and relaxation vanish after the decay of the optical pulse and $\mathrm{e}-\mathrm{h}$ polarizations.

Eq.(41) predicts an extremely fast response of the spin system, determined by the effective magnetic field pulse $\mathbf{h}(t)$, whose speed is limited only by the pulse duration. This result points to a new way of controlling the magnetization during femtosecond time scales, much shorter than those accomplished so far. The last two terms on the rhs of Eq.(41) demonstrate an ultrafast modification of the effective magnetic field $\mathbf{H}$. Eq. (41) therefore predicts two regimes of time evolution, which can be separated due to their very different timescales. The initial temporal regime is controlled by the pulsed field $\mathbf{h}(t)$ and vanishes soon after the optical excitation. The second temporal regime is controlled by the anisotropy field $\mathbf{H}(t)$, Eq. (40), due to the thermal Fermi sea, which depends nonlinearly on $\mathbf{S}(t)$.

We now turn to the effective light-induced magnetic field pulse $\mathbf{h}(t)$. As can be seen from Eq.(41), in order to trigger magnetization dynamics, $\mathbf{h}(t)$ must have a component perpendicular to the ground state $\mathrm{Mn}$ spin and the easy axis. The direction of $\mathbf{h}(t)$ is determined by the optical transition selection rules and by the interband polarizations. Figs 8 and 9 show the three components $\mathbf{h}_{x}(t), \mathbf{h}_{y}(t)$, and $\mathbf{h}_{z}(t)$ in the case of strong spin dephas- (a)

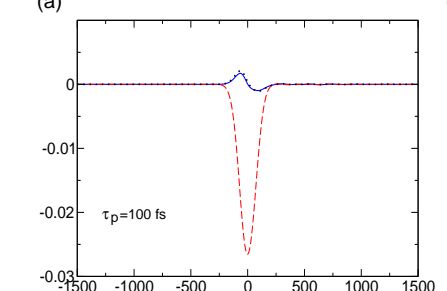

(b)
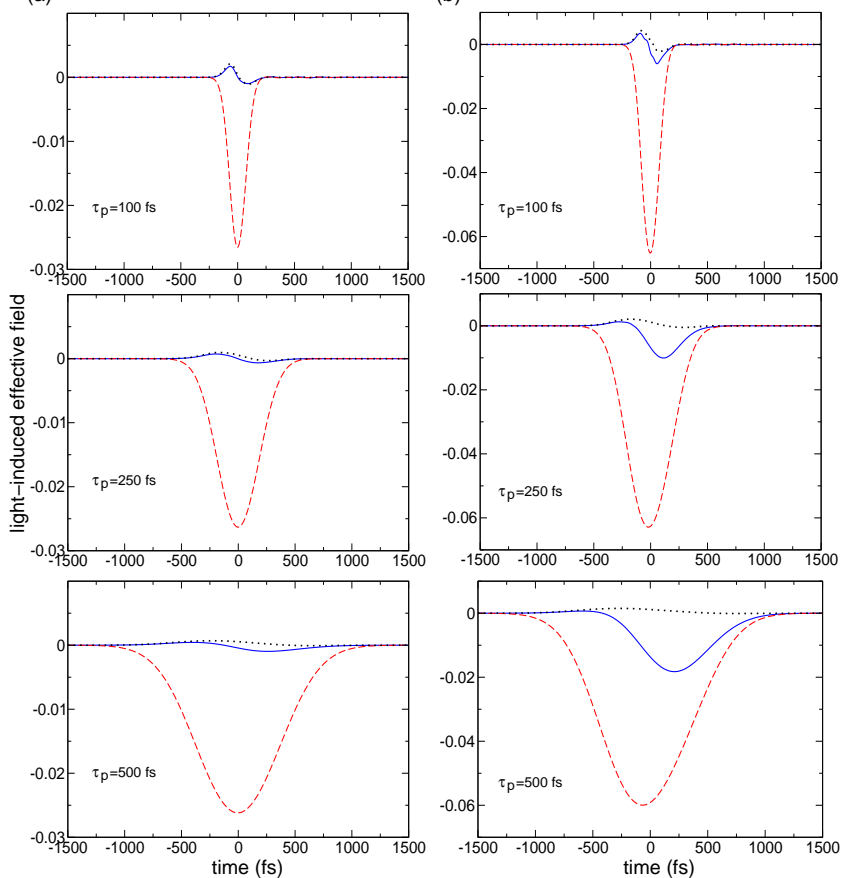

FIG. 9: (Color online) Same as Fig 8 but with $\Gamma_{\perp}=0$, $T_{2}=330 \mathrm{fs}$.

ing (Fig 8) or uncoupled hole spin states (Fig 9). It is clear by comparing the two figures that the direction of $\mathbf{h}(t)$, which governs the Mn spin trajectory, depends on the dephasing. The mosty striking effect of $\Gamma_{\perp}$ is the development, on the time scale of the optical excitation, of a large component $\mathbf{h}_{z}$ in the direction of optical pulse propagation. This component is strongly suppressed in the case of weak dephasing. As can be seen from Eq.(41), $\mathbf{h}_{z}$ rotates the $\mathrm{Mn}$ spin within the $\mathrm{x}-\mathrm{y}$ plane, in a counterclockwise direction, and leads to spin relaxation towards the positive $\mathrm{z}$-axis, i.e. to a magnetization tilt out of the $\mathrm{x}-\mathrm{y}$ plane. On the other hand, for $\Gamma_{\perp}=0$, the Gilbert damping coefficient vanishes, $\alpha=0$, which suppresses the light-induced Mn spin rotation. The only effect of the photoexcitation is then the relaxation described by the last term of Eq. (41), which causes the Mn spin to relax towards the negative $\mathrm{y}$-axis (counter-clockwise rotation within the $x-y$ plane) since $\mathbf{h}_{z} \approx 0$ in this case. The above trends are consistent with the full numerical calculation of the mean field equations discussed in the previous section. In Appendix E we derive an equation of motion for $\mathbf{h}(t)$ that demonstrates its dependence on the optical transition selection rules, the hole spin polarization, the magnetic exchange interaction, and the hole spin dephasing.

\section{CONCLUSIONS}

In conclusion, we presented a theory of nonlinear spin dynamics in ferromagnetic semiconductors and demon- 
strated the main effects common in all such systems by considering a single spin-degenerate electron and hole band. Our main result of relevance to experiments is the existence of two different temporal regimes of magnetization dynamics. The initial regime lasts for time scales comparable to the optical pulse duration. It is governed by magnetization precession and relaxation around an effective magnetic field pulse generated by the optical excitation via second--order coherent nonlinear processes. The second temporal regime is governed by the magnetic anisotropy due to the thermal carriers. We showed that the shape of the magnetization trajectory depends sensitively on the hole spin dephasing, the magnetic easy axes, the transition matrix elements, and the optical pulse intensity, duration, and helicity. The latter determine the magnitude and direction of the ultrafast magnetization tilt from the magnetic easy axis in response to the photoexcitation of hole spin, which in turn determines the importance of nonlinear magnetic effects during many picoseconds. To interpret our numerical results, we derived an effective Landau-Gilbert-like equation of motion. This equation shows that an effective magnetic field pulse, generated by the interband optical polarizations via a Raman-like process, triggers precession and relaxation during femtosecond time scales. This magnetic field pulse may be thought of as an ultrafast correction to the anisotropy field. The picture of ultrafast magnetization dynamics conveyed by our results and, in particular, the existence of two temporal regimes is consistent with the recent experimental observation of a light-induced modification of the magnetic anisotropy field in $\mathrm{Ga}(\mathrm{Mn}) \mathrm{As}$ during femtosecond timesales, induced by nonthermal high energy carriers ${ }^{35}$ A more detailed comparison between theory and experiment must be performed in the future. Our calculations point out the need for further experiments in order to explore the control of the magnetization trajectory by changing the optical excitation intensity, duration, central frequency, and helicity. For a complete understanding of the magnetization dynamics in ferromagnetic semiconductors, the role of fluctuations and carrier-spin correlations,, 54 the bandstructure, and disorder must also be considered.

This work was supported by the EU STREP program HYSWITCH.

\section{APPENDIX A}

In this Appendix we derive the relaxation and dephasing contributions to the hole spin equations of motion. The hole spin is expressed in the basis of hole spin eigenstates as

$$
\begin{array}{r}
\mathbf{s}_{\mathbf{k} m z}^{h}=\frac{1}{2}\left[\left\langle\hat{h}_{-\mathbf{k} m \uparrow}^{\dagger} \hat{h}_{-\mathbf{k} m \uparrow}\right\rangle-\left\langle\hat{h}_{-\mathbf{k} m \downarrow}^{\dagger} \hat{h}_{-\mathbf{k} m \downarrow}\right\rangle\right], \\
\mathbf{s}_{\mathbf{k} m+}^{h}=\mathbf{s}_{\mathbf{k} m x}^{h}+i \mathbf{s}_{\mathbf{k} m y}^{h}=\left\langle\hat{h}_{-\mathbf{k} m \uparrow}^{\dagger} \hat{h}_{-\mathbf{k} m \downarrow}\right\rangle .
\end{array}
$$

From Eqs.(19) and (20) we obtain for the corresponding hole density matrices after using straightforward algebra and the property $\hat{h}^{2}=0$ :

$$
\left.\partial_{t}\left\langle\hat{h}_{-\mathbf{k} m \Downarrow}^{\dagger} \hat{h}_{-\mathbf{k} m \Uparrow}\right\rangle\right|_{\text {relax }}=-\frac{\Gamma_{\perp}}{2}\left\langle\hat{h}_{-\mathbf{k} m \Downarrow}^{\dagger} \hat{h}_{-\mathbf{k} m \Uparrow}\right\rangle
$$

By choosing the $\mathrm{z}^{-}$-axis parallel to the Mn spin and noting the expression Eq. (A2), we obtain Eq. (23) that describes the dephasing of the hole spin component $\mathbf{s}_{\mathbf{k} m \perp}^{h}$ perpendicular to the Mn spin. Similarly we obtain from Eqs. (19) and (20)

$$
\begin{aligned}
& \left.\partial_{t}\left\langle\hat{h}_{-\mathbf{k} m \Downarrow}^{\dagger} \hat{h}_{-\mathbf{k} m \Downarrow}\right\rangle\right|_{\text {relax }}=-\left.\partial_{t}\left\langle\hat{h}_{-\mathbf{k} m \Uparrow}^{\dagger} \hat{h}_{-\mathbf{k} m \Uparrow}\right\rangle\right|_{\text {relax }} \\
& =\Gamma_{\perp}\left[\left\langle\hat{h}_{-\mathbf{k} m \Uparrow}^{\dagger} \hat{h}_{-\mathbf{k} m \Uparrow}\right\rangle\right. \\
& \left.-\left\langle\hat{h}_{-\mathbf{k} m \Uparrow}^{\dagger} \hat{h}_{-\mathbf{k} m \Downarrow}^{\dagger} \hat{h}_{-\mathbf{k} m \Downarrow} \hat{h}_{-\mathbf{k} m \Uparrow}\right\rangle\right],
\end{aligned}
$$

which conserve the total hole population

$$
N_{\mathbf{k} m}^{h}=\left\langle\hat{h}_{-\mathbf{k} m \Downarrow}^{\dagger} \hat{h}_{-\mathbf{k} m \Downarrow}\right\rangle+\left\langle\hat{h}_{-\mathbf{k} m \Uparrow}^{\dagger} \hat{h}_{-\mathbf{k} m \Uparrow}\right\rangle .
$$

Using the factorization

$$
\left\langle\hat{h}_{1}^{\dagger} \hat{h}_{2}^{\dagger} \hat{h}_{3} \hat{h}_{4}\right\rangle=\left\langle\hat{h}_{1}^{\dagger} \hat{h}_{4}\right\rangle\left\langle\hat{h}_{2}^{\dagger} \hat{h}_{3}\right\rangle-\left\langle\hat{h}_{1}^{\dagger} \hat{h}_{3}\right\rangle\left\langle\hat{h}_{2}^{\dagger} \hat{h}_{4}\right\rangle
$$

and the relation (obtained from Eqs. (A1) and (A5)

$$
\left\langle\hat{h}_{-\mathbf{k} m \sigma}^{\dagger} \hat{h}_{-\mathbf{k} m \sigma}\right\rangle=N_{\mathbf{k} m}^{h} / 2+\sigma s_{\mathbf{k} m \|}^{h}
$$

where $\sigma= \pm 1$, we obtain Eq.(24) for the hole spin relaxation.

\section{APPENDIX B}

In this appendix we derive the polarization dephasing $\left.\partial_{t} P_{\mathbf{k} m n \sigma}\right|_{\text {relax }}$ by using Eqs.(19) and (20). After straightforward algebra and using the properties $L_{\mathbf{k} m}^{\dagger} \hat{h}_{\mathbf{k} m \sigma} L_{\mathbf{k} m}=0, \hat{h}^{2}=0$, we obtain after noting that the terms in the summation of Eq. (19) vanish unless $\left(\mathbf{k}^{\prime} m^{\prime}\right)=(\mathbf{k} m)$ :

$$
\begin{aligned}
\left.\partial_{t} P_{\mathbf{k} m n \sigma}\right|_{\text {relax }}= & -\frac{\Gamma_{\perp}}{2}\left\langle\hat{h}_{-\mathbf{k} m \Uparrow}^{\dagger} \hat{h}_{-\mathbf{k} m \Uparrow} \hat{h}_{-\mathbf{k} m \sigma} \hat{e}_{\mathbf{k} n}\right\rangle \\
& -\frac{\Gamma_{\perp}}{2}\left\langle\hat{h}_{-\mathbf{k} m \sigma} \hat{e}_{\mathbf{k} n} \hat{h}_{-\mathbf{k} m \Downarrow} \hat{h}_{-\mathbf{k} m \Downarrow}^{\dagger}\right\rangle .
\end{aligned}
$$

We now note the relation between the hole operators with spin along the $\mathrm{z}$-axis of pulse propagation or along the Mn spin direction:

$$
\begin{gathered}
\hat{h}_{\mathbf{k} m \Uparrow}=e^{i \phi / 2} \cos \frac{\theta}{2} \hat{h}_{\mathbf{k} m \uparrow}+e^{-i \phi / 2} \sin \frac{\theta}{2} \hat{h}_{\mathbf{k} m \downarrow}, \\
\hat{h}_{\mathbf{k} m \Downarrow}=-e^{i \phi / 2} \sin \frac{\theta}{2} \hat{h}_{\mathbf{k} m \uparrow}+e^{-i \phi / 2} \cos \frac{\theta}{2} \hat{h}_{\mathbf{k} m \downarrow}
\end{gathered}
$$

where $\theta(t)$ and $\phi(t)$ are the polar coordinates that define the direction of the Mn spin $\mathbf{S}(t)$ and the operators $\hat{h}_{\mathbf{k} m \uparrow}$ 
and $\hat{h}_{\mathbf{k} m \downarrow}$ create hole states with spin along the $\mathrm{z}^{-}$-axis. Using Eqs. (B2) and (B3), the property $\hat{h}^{2}=0$, and the relations $\mathbf{S}^{+} / S=e^{i \phi} \sin \theta, \mathbf{S}_{z} / S=\cos \theta$ we obtain from Eq.(B1) after straightforward algebra

$$
\begin{aligned}
& \left.\partial_{t} P_{\mathbf{k} m n \uparrow}\right|_{\text {relax }}= \\
& -\Gamma_{\perp}\left[\frac{1-\mathbf{S}_{z} / S}{2}\left\langle\hat{h}_{-\mathbf{k} m \downarrow}^{\dagger} \hat{h}_{-\mathbf{k} m \downarrow} \hat{h}_{-\mathbf{k} m \uparrow} \hat{e}_{\mathbf{k} n \downarrow}\right\rangle\right. \\
& \left.+\frac{\mathbf{S}_{-}}{2 S}\left\langle\hat{h}_{-\mathbf{k} m \uparrow}^{\dagger} \hat{h}_{-\mathbf{k} m \downarrow} \hat{h}_{-\mathbf{k} m \uparrow} \hat{e}_{\mathbf{k} n \downarrow}\right\rangle\right] \\
& -\Gamma_{\perp}\left[\frac{1+\mathbf{S}_{z} / S}{2}\left\langle\hat{h}_{-\mathbf{k} m \uparrow} \hat{e}_{\mathbf{k} n \downarrow} \hat{h}_{-\mathbf{k} m \downarrow} \hat{h}_{-\mathbf{k} m \downarrow}^{\dagger}\right\rangle\right. \\
& \left.-\frac{\mathbf{S}_{-}}{2 S}\left\langle\hat{h}_{-\mathbf{k} m \uparrow} \hat{e}_{\mathbf{k} n \downarrow} \hat{h}_{-\mathbf{k} m \downarrow} \hat{h}_{-\mathbf{k} m \uparrow}^{\dagger}\right\rangle\right]
\end{aligned}
$$

and

$$
\begin{aligned}
& \left.\partial_{t} P_{\mathbf{k} m n \downarrow}\right|_{\text {relax }}= \\
& -\Gamma_{\perp}\left[\frac{1+\mathbf{S}_{z} / S}{2}\left\langle\hat{h}_{-\mathbf{k} m \uparrow}^{\dagger} \hat{h}_{-\mathbf{k} m \uparrow} \hat{h}_{-\mathbf{k} m \downarrow} \hat{e}_{\mathbf{k} n \downarrow}\right\rangle\right. \\
& \left.+\frac{\mathbf{S}_{+}}{2 S}\left\langle\hat{h}_{-\mathbf{k} m \downarrow}^{\dagger} \hat{h}_{-\mathbf{k} m \uparrow} \hat{h}_{-\mathbf{k} m \downarrow} \hat{e}_{\mathbf{k} n \downarrow}\right\rangle\right] \\
& -\Gamma_{\perp}\left[\frac{1-\mathbf{S}_{z} / S}{2}\left\langle\hat{h}_{-\mathbf{k} m \downarrow} \hat{e}_{\mathbf{k} n \downarrow} \hat{h}_{-\mathbf{k} m \uparrow} \hat{h}_{-\mathbf{k} m \uparrow}^{\dagger}\right\rangle\right. \\
& \left.-\frac{\mathbf{S}_{+}}{2 S}\left\langle\hat{h}_{-\mathbf{k} m \downarrow} \hat{e}_{\mathbf{k} n \downarrow} \hat{h}_{-\mathbf{k} m \uparrow} \hat{h}_{-\mathbf{k} m \downarrow}^{\dagger}\right\rangle\right] .
\end{aligned}
$$

We now factorize the higher density matrices in the above equations as follows:

$$
\begin{aligned}
& \left\langle\hat{h}_{1}^{\dagger} \hat{h}_{2} \hat{h}_{3} \hat{e}\right\rangle=\left\langle\hat{h}_{1}^{\dagger} \hat{h}_{2}\right\rangle\left\langle\hat{h}_{3} \hat{e}\right\rangle-\left\langle\hat{h}_{1}^{\dagger} \hat{h}_{3}\right\rangle\left\langle\hat{h}_{2} \hat{e}\right\rangle, \\
& \left\langle\hat{h}_{3} \hat{e} \hat{h}_{2} \hat{h}_{1}^{\dagger}\right\rangle=\left\langle\hat{h}_{3} \hat{e}\right\rangle\left\langle\hat{h}_{2} \hat{h}_{1}^{\dagger}\right\rangle-\left\langle\hat{h}_{2} \hat{e}\right\rangle\left\langle\hat{h}_{3} \hat{h}_{1}^{\dagger}\right\rangle .
\end{aligned}
$$

We thus obtain that

$$
\begin{aligned}
& \left.\partial_{t} P_{\mathbf{k} m n \uparrow}\right|_{\text {relax }}= \\
& -\Gamma_{\perp}\left\{\frac{1-\mathbf{S}_{z} / S}{2}\left(n_{\mathbf{k} m \downarrow}^{h} P_{\mathbf{k} m n \uparrow}-\mathbf{s}_{\mathbf{k} m-}^{h} P_{\mathbf{k} m n \downarrow}\right)\right. \\
& +\frac{\mathbf{S}_{-}}{2 S}\left(\mathbf{s}_{\mathbf{k} m+}^{h} P_{\mathbf{k} m n \uparrow}-n_{\mathbf{k} m \uparrow}^{h} P_{\mathbf{k} m n \downarrow}\right) \\
& +\frac{1+\mathbf{S}_{z} / S}{2}\left[P_{\mathbf{k} m n \uparrow}\left(1-n_{\mathbf{k} m \downarrow}^{h}\right)+P_{\mathbf{k} m n \downarrow} \mathbf{s}_{\mathbf{k} m-}^{h}\right] \\
& \left.+\frac{\mathbf{S}_{-}}{2 S}\left[\mathbf{s}_{\mathbf{k} m+}^{h} P_{\mathbf{k} m n \uparrow}+P_{\mathbf{k} m n \downarrow}\left(1-n_{\mathbf{k} m \uparrow}^{h}\right)\right]\right\} .
\end{aligned}
$$

and

$$
\begin{aligned}
& \left.\partial_{t} P_{\mathbf{k} m n \downarrow}\right|_{\text {relax }}= \\
& -\Gamma_{\perp}\left\{\frac{1+\mathbf{S}_{z} / S}{2}\left(n_{\mathbf{k} m \uparrow}^{h} P_{\mathbf{k} m n \downarrow}-\mathbf{s}_{\mathbf{k} m+}^{h} P_{\mathbf{k} m n \uparrow}\right)\right. \\
& +\frac{\mathbf{S}_{+}}{2 S}\left(\mathbf{s}_{\mathbf{k} m-}^{h} P_{\mathbf{k} m n \downarrow}-n_{\mathbf{k} m \downarrow}^{h} P_{\mathbf{k} m n \uparrow}\right) \\
& +\frac{1-\mathbf{S}_{z} / S}{2}\left[P_{\mathbf{k} m n \downarrow}\left(1-n_{\mathbf{k} m \uparrow}^{h}\right)+P_{\mathbf{k} m n \uparrow} \mathbf{s}_{\mathbf{k} m+}^{h}\right] \\
& \left.+\frac{\mathbf{S}_{+}}{2 S}\left[\mathbf{s}_{\mathbf{k} m-}^{h} P_{\mathbf{k} m n \downarrow}+P_{\mathbf{k} m n \uparrow}\left(1-n_{\mathbf{k} m \downarrow}^{h}\right)\right]\right\},
\end{aligned}
$$

where $n_{\mathbf{k} m \sigma}$ are the spin-polarized hole populations. After expressing the latter by using Eq. (A7) we obtain after some algebra the polarization dephasing contributions Eqs. (27) and (28).

\section{APPENDIX C}

In this appendix we derive an effectve equation of motion for the Mn spin by expanding around the adiabatic limit. First we use the hole spin decomposition Eq.(21) into a component parallel $\hat{\mathbf{S}}(t)\left(s_{\mathbf{k} \|}^{h}\right)$ and a component perpendicular to $\hat{\mathbf{S}}(t)\left(\mathbf{s}_{\mathbf{k} \perp}^{h}\right)$. The unadiabatic contribution $\mathbf{s}_{\mathbf{k} \perp}^{h}$ is responsible for triggering the Mn spin precession. After substituting Eq.(21) into Eq.(15) and noting that the Mn spin magnitude $S$ remains constant in time we obtain

$$
\begin{gathered}
\partial_{t} \mathbf{s}_{\mathbf{k} \perp}^{h}=\beta c \mathbf{S} \times \mathbf{s}_{\mathbf{k} \perp}^{h}+\operatorname{Im} \mathbf{h}_{\mathbf{k}}(t)-s_{\mathbf{k} \|}^{h} \partial_{t} \hat{\mathbf{S}} \\
-\hat{\mathbf{S}}\left[\partial_{t} s_{\mathbf{k} \|}^{h}+\Gamma_{\|}\left(s_{\mathbf{k} \|}^{h}+m_{\mathbf{k}}^{h}\right)\right]-\Gamma_{\perp} \mathbf{s}_{\mathbf{k} \perp}^{h} .
\end{gathered}
$$

By projecting out the components parallel and perpendicular to $\hat{\mathbf{S}}$ and using the relations $\mathbf{S} \cdot \partial_{t} \mathbf{S}=\partial_{t} S^{2} / 2=0$ and $\mathbf{s}_{\mathbf{k} \perp}^{h} \cdot \mathbf{S}=0$ we obtain that

$$
\partial_{t} s_{\mathbf{k} \|}^{h}+\Gamma_{\|}\left(s_{\mathbf{k} \|}^{h}+m_{\mathbf{k}}^{h}\right)=\operatorname{Im} h_{\mathbf{k} \|}(t)+\mathbf{s}_{\mathbf{k} \perp}^{h} \cdot \partial_{t} \hat{\mathbf{S}}
$$

and

$$
\left.\left.\partial_{t} \mathbf{s}_{\mathbf{k} \perp}^{h}\right|_{\perp}=\beta c \mathbf{S} \times \mathbf{s}_{\mathbf{k} \perp}^{h}+\operatorname{Im} \mathbf{h}_{\mathbf{k} \perp}-\Gamma_{\perp} \mathbf{s}_{\mathbf{k} \perp}^{h}-s_{\mathbf{k} \|}^{h} \partial_{t} \hat{\mathbf{S}} \mathrm{C} 3\right)
$$

We now focus on the perpendicular component $\mathbf{s}_{\perp}^{h}$. We note from Eq.(8) that the motion of $\mathbf{S}(t)$ is characterized by a precession frequency $\omega$ determined by the field $\mathbf{H}$ and the mean hole spin $\beta \mathbf{s}_{\perp}^{h}$ :

$$
\partial \hat{\mathbf{S}}=\omega \times \hat{\mathbf{S}}, \omega=\beta \mathbf{s}_{\perp}^{h}-\mathbf{H}
$$

where

$$
\mathbf{s}^{h}=\frac{1}{V} \sum_{\mathbf{k}} \mathbf{s}_{\mathbf{k}}^{h}
$$

is the mean hole spin. In III-Mn-V semiconductors, this precession is much slower than the motion of the hole spin $\mathbf{s}_{\perp}^{h}$, which is characterized by the precession energy $\beta c S$ and the relaxation rate $\Gamma_{\perp}$. When considering the slower Mn spin dynamics, we can then substitute $\mathbf{s}_{\perp}^{h}$ by its steady state value in the frame of reference that rotates with the Mn spin and $\hat{\mathbf{S}}(t)$. The rate of change of any vector $\mathbf{A}$ as seen by an observer in the rotating frame, $\left.\partial_{t} \mathbf{A}\right|_{\text {rot }}$, is related to the corresponding rate of change in the inertial frame, $\partial_{t} \mathbf{A}$, by

$$
\partial_{t} \mathbf{A}=\left.\partial_{t} \mathbf{A}\right|_{\text {rot }}+\omega \times \mathbf{A} .
$$

We thus obtain in the case of the hole spin

$$
\partial_{t} \mathbf{s}_{\perp}^{h}=\left.\partial_{t} \mathbf{s}_{\perp}^{h}\right|_{\text {rot }}-\mathbf{H} \times \mathbf{s}_{\perp}^{h} .
$$


Projecting in the direction perpendicular to the Mn spin using Eq.(22) we obtain that

$$
\left.\partial_{t} \mathbf{s}_{\mathbf{k} \perp}^{h}\right|_{\perp}=\left.\partial_{t} \mathbf{s}_{\perp}^{h}\right|_{r o t, \perp}+H_{\|} \frac{\mathbf{s}_{\perp}^{h} \times \mathbf{S}}{S},
$$

where we used the vector property

$$
\mathbf{A} \times(\mathbf{B} \times \mathbf{C})=(\mathbf{A} \cdot \mathbf{C}) \mathbf{B}-(\mathbf{A} \cdot \mathbf{B}) \mathbf{C} .
$$

The rotating frame approximation corresponds to neglecting $\left.\partial_{t} \mathbf{s}_{\perp}^{h}\right|_{\text {rot, },}$.

To obtain an effective equation of motion for the $\mathrm{Mn}$ spin, we use Eq. (8) for $\mathbf{s}_{\perp}^{h} \times \mathbf{S}$ to eliminate the hole spin $\mathbf{s}_{\perp}^{h}$, defined by Eqs (C5) and (22), from Eq.(C3):

$$
\mathbf{s}_{\perp}^{h}=\hat{\mathbf{S}} \times\left(\mathbf{s}_{\perp}^{h} \times \hat{\mathbf{S}}\right)=\frac{\hat{\mathbf{S}} \times \partial_{t} \hat{\mathbf{S}}+\hat{\mathbf{S}} \times(\mathbf{H} \times \hat{\mathbf{S}})}{\beta} .
$$

By summing Eq. C33 over all momenta we thus obtain within the rotating frame approximation after using the definition Eq.(42) that

$$
\begin{aligned}
& \left(\beta c S+\beta s_{\|}^{h}+H_{\|}\right) \partial_{t} \hat{\mathbf{S}}=\left(\beta c S+H_{\|}\right) \hat{\mathbf{S}} \times \mathbf{H} \\
& +\beta \hat{\mathbf{S}} \times(\mathbf{h} \times \hat{\mathbf{S}})+\Gamma_{\perp} \hat{\mathbf{S}} \times\left(\hat{\mathbf{S}} \times \mathbf{H}-\partial_{t} \hat{\mathbf{S}}\right) .
\end{aligned}
$$

The above nonlinear equation of motion is governed by the magnetic field $\mathbf{H}$, the effective magnetic field $\mathbf{h}$ determined by the interband $\mathrm{e}-\mathrm{h}$ polarizations, and the hole spin relaxation $\left(\Gamma_{\|}\right)$and dephasing $\left(\Gamma_{\perp}\right)$ rates. Eq. (C11) can be transformed into an effective time-dependent Landau-Gilbert-like equation of motion by taking the cross product of both sides with $\hat{\mathbf{S}}$ and using the vector property Eq.(C9) and the property $\mathbf{S} \cdot \partial_{t} \mathbf{S}=0$ :

$$
\begin{array}{lr}
\left(\beta c S+\beta s_{\|}^{h}+H_{\|}\right) \partial_{t} \hat{\mathbf{S}} \times \hat{\mathbf{S}}=\left(\beta c S+H_{\|}\right) \hat{\mathbf{S}} \times(\mathbf{H} \times \hat{\mathbf{S}}) \\
+\beta \mathbf{h} \times \hat{\mathbf{S}}+\Gamma_{\perp} \hat{\mathbf{S}} \times \mathbf{H}-\Gamma_{\perp} \partial_{t} \hat{\mathbf{S}} .
\end{array}
$$

Substituting the above expression into Eq. (C11) we obtain Eq.(41).

\section{APPENDIX D}

In this appendix we derive the relaxation of the hole spin component $s_{\|}^{h}$ parallel to the Mn spin $\mathbf{S}(t)$, determined by Eq. (C2). We eliminate $\mathbf{s}_{\perp}^{h}$ from Eq. (C2) by using the property $\mathbf{s}_{\perp}^{h} \cdot \partial_{t} \hat{\mathbf{S}}=\mathbf{H} \cdot \partial_{t} \hat{\mathbf{S}} / \beta$, obtained after using the properties $\left(\overline{\mathbf{S}} \times \partial_{t} \mathbf{S}\right) \cdot \partial_{t} \mathbf{S}=0, \mathbf{S} \cdot \partial_{t} \mathbf{S}=\partial_{t} S^{2}=0$, and some algebra. We also eliminate $\partial_{t} \mathbf{S}$ from Eq.(C2) by projecting both sides of Eq.(41) with $\mathbf{H}$ and using the vector property $\mathbf{A} \cdot(\mathbf{B} \times \mathbf{C})=\mathbf{B} \cdot(\mathbf{C} \times \mathbf{A})$ and the relations $\mathbf{H}^{2}=H_{\|}^{2}+H_{\perp}^{2}$ and $\mathbf{h} \cdot \mathbf{H}=h_{\|} H_{\|}+\mathbf{h}_{\perp} \cdot \mathbf{H}_{\perp}$. By summing both sides of Eq. (C2) over all momenta we finally obtain the equation of motion

$$
\begin{gathered}
\partial_{t} s_{\|}^{h}+\Gamma_{\|}\left(s_{\|}^{h}+m_{h}\right)+\frac{\Gamma_{\perp} \mathbf{H}_{\perp}^{2}+\beta \mathbf{H}_{\perp} \cdot \mathbf{h}_{\perp}}{\left(\beta c S+\beta s_{\|}^{h}+H_{\|}\right)^{2}+\Gamma_{\perp}^{2}} s_{\|}^{h} \\
=h_{\|}+\frac{\Gamma_{\perp}(\mathbf{H} \times \mathbf{h})_{\|}-\left(\beta c S+H_{\|}\right) \mathbf{H}_{\perp} \cdot \mathbf{h}_{\perp}}{\left(\beta c S+\beta s_{\|}^{h}+H_{\|}\right)^{2}+\Gamma_{\perp}^{2}} . \quad(\mathrm{D} 1)
\end{gathered}
$$

\section{APPENDIX E}

In this appendix we derive the equation of motion that determines the time evolution of the effective magentic field pulse $\mathbf{h}(t)=\mathcal{E}(t) \hat{\mathbf{h}}(t)$ that governs the intial femtosecond magnetization re-orientation and relaxation. Using the equations of motion of the interband polarizations, Eqs.(31), (32), (34), and (33), we obtain the equation of motion

$$
i \partial_{t} \hat{\mathbf{h}}_{\mathbf{k}}=\Omega_{\mathbf{k}} \hat{\mathbf{h}}_{\mathbf{k}}+\frac{i}{2} \boldsymbol{\Delta}_{\mathbf{k}} \times \hat{\mathbf{h}}_{\mathbf{k}}+\frac{\boldsymbol{\Delta}_{\mathbf{k}} p_{\mathbf{k}}}{2}+\mathcal{E}(t) \mathcal{S}_{\mathbf{k}},
$$

where $\Omega_{\mathbf{k}}$ and $\boldsymbol{\Delta}_{\mathbf{k}}$ were defined by Eqs.(35) and (36),

$$
p_{\mathbf{k}}(t)=\mu_{+}^{*} P_{\mathbf{k} \uparrow}^{+}(t)+\mu_{-}^{*} P_{\mathbf{k} \downarrow}^{-}(t)
$$

satisfies the equation of motion

$$
i \partial_{t} p_{\mathbf{k}}=\Omega_{\mathbf{k}} p_{\mathbf{k}}+\frac{\boldsymbol{\Delta}_{\mathbf{k}} \cdot \mathbf{h}_{\mathbf{k}}}{2}-\mathcal{E}(t) \mathcal{N}_{\mathbf{k}}
$$

where

$$
\begin{array}{r}
\mathcal{N}_{\mathbf{k}}=\left(\left|\mu_{+}\right|^{2}+\left|\mu_{-}\right|^{2}\right)\left[1-\left(N_{\mathbf{k}}^{e}+N_{\mathbf{k}}^{h}\right) / 2\right] \\
-\left(\left|\mu_{+}\right|^{2}-\left|\mu_{-}\right|^{2}\right)\left(s_{\mathbf{k} z}^{h}-s_{\mathbf{k} z}^{e}\right),
\end{array}
$$

and

$$
\begin{aligned}
& \mathcal{S}_{\mathbf{k} x}=\left(\left|\mu_{+}\right|^{2}+\left|\mu_{-}\right|^{2}\right) s_{\mathbf{k} x}^{h}+i\left(\left|\mu_{+}\right|^{2}-\left|\mu_{-}\right|^{2}\right) s_{\mathbf{k} y}^{h} \\
& +2 \operatorname{Re}\left(\mu_{+} \mu_{-}^{*}\right) s_{\mathbf{k} x}^{e}+2 \operatorname{Im}\left(\mu_{+} \mu_{-}^{*}\right) s_{\mathbf{k} y}^{e} \\
& \mathcal{S}_{\mathbf{k} y}=\left(\left|\mu_{+}\right|^{2}+\left|\mu_{-}\right|^{2}\right) s_{\mathbf{k} y}^{h}-i\left(\left|\mu_{+}\right|^{2}-\left|\mu_{-}\right|^{2}\right) s_{\mathbf{k} x}^{h} \\
& +2 \operatorname{Re}\left(\mu_{+} \mu_{-}^{*}\right) s_{\mathbf{k} y}^{e}-2 \operatorname{Im}\left(\mu_{+} \mu_{-}^{*}\right) s_{\mathbf{k} x}^{e} \\
& \mathcal{S}_{\mathbf{k} z}=-\left(\left|\mu_{+}\right|^{2}-\left|\mu_{-}\right|^{2}\right)\left[1-\left(N_{\mathbf{k}}^{h}+N_{\mathbf{k}}^{e}\right) / 2\right] \\
& +\left(\left|\mu_{+}\right|^{2}+\left|\mu_{-}\right|^{2}\right)\left(s_{\mathbf{k} z}^{h}-\mathbf{s}_{\mathbf{k} z}^{e}\right) .
\end{aligned}
$$

We note from the above equations that, in the absence of spin polarization in the ground state, $\mathbf{S}=\mathbf{s}^{h}=\mathbf{s}^{e}=0$, $\mathbf{h}(t)$ points along the $\mathrm{z}$-direction of optical field propagation. On the other hand, in the presence of spinpolarized holes in the ground state as in ferromagnetic semiconductors, $\mathbf{h}$ develops additional components determined by $\mathbf{s}^{h}$, the Mn spin and exchange interaction $\boldsymbol{\Delta}_{\mathbf{k}}$, and by the magnetic anisotropy. 
1 E. L. Nagaev, Phys. Rep. 346, 387 (2001).

2 See e.g. Colossal Magnetoresistance Oxides, ed. Y. Tokura (Gordon Breach, Singapore, 2000).

3 H. Ohno, Science 281, 951 (1998).

4 T. Dietl, H. Ohno, F. Matsukura, J. Cibert, and D. Ferrand, Science 287, 1019 (2000).

5 T. Jungwirth, J. Sinova, J. Masek, J. Kucera, amd A. H. MacDonald, Rev. Mod. Phys. 78, 809 (2006).

6 S. A. Wolf, D. D. Awschalom, R. A. Buhrman, J. M. Daughton, S. von Molnar, M. L. Roukes, A. Y. Chtchelkanova, and D. M. Treger, Science 294, 1488 (2001).

7 J. Wang, C. Sun, Y. Hashimoto, J. Kono, G. A. Khodaparast, L. Cywinski, L. J. Sham, G. D. Sanders, C. Stanton, and H. Munekata, J. Phys. Cond. Matt. 18, R501 (2006).

8 E. Beaurepaire, J.-C. Merle, A. Daunois, and J.-Y. Bigot, Phys. Rev. Lett. 76, 4250 (1996).

9 L. Guidoni, E. Beaurepaire, and J.-Y. Bigot, Phys. Rev. Lett. 89, 017401 (2002).

10 J.-Y. Bigot, L. Guidoni, E. Beaurepaire, and P. N. Saeta, Phys. Rev. Lett. 93, 077401 (2004).

11 B. Koopmans, M. van Kampen, J. T. Kohlhepp, and W. J.M. de Jonge, Phys. Rev. Lett. 85, 844 (2000).

12 B. Koopmans, J. J. M. Ruigrok, F. Dalla Longa, and W. J. M. de Jonge, Phys. Rev. Lett. 95, 267207 (2005).

13 E. Kojima, R. Shimano, Y. Hashimoto, S. Katsumoto, Y. Iye, and M. Kuwata-Gonokami, Phys. Rev. B 68, 193203 (2003).

14 J. Wang, C. Sun, J. Kono, A. Oiwa, H. Munekata, L. Cywinski, and L. J. Sham, Phys. Rev. Lett. 95, 167401 (2005)

15 J.-Y. Bigot, M. Vomir, L. H. F. Andrade, and E. Beaurepaire, Chem. Phys. 318, 137 (2005).

16 M. Vomir, L. H. F. Andrade, L. Guidoni, E. Beaurepaire, and J.-Y. Bigot, Phys. Rev. Lett. 94, 237601 (2005).

17 M. van Kampen, C. Jozsa, J. T. Kohlhepp, P. LeClair, L. Lagae, W. J. M. de Jonge, and B. Koopmans, Phys. Rev. Lett. 88, 227201 (2002).

18 A. V. Kimel, A. Kirilyuk, A. Tsvetkov, R. V. Pisarev, and Th. Rasing, Nature 429, 850 (2004).

19 H. Takechi, A. Oiwa, K. Nomura, T. Kondo, and H. Munekata, Phys. Stat. Sol. (c) 3, 4267 (2006).

20 A. Oiwa, H. Takechi, and H. Munekata, J. Supercond. 18, 9 (2005).

21 J. Qi, Y. Xu, N. H. Tolk, X. Liu, J. K. Furdyna, and I. E. Perakis, Appl. Phys. Lett. 91, 112506 (2007).

22 A. V. Kimel, A. Kirilyuk, O. A. Usachev, R. V. Pisarev, A. M. Balbashov, and Th. Rasing, Nature 435, 655 (2005).

${ }^{23}$ F. Hansteen, A. Kimel, A. Kirilyuk, and Th. Rasing, Phys. Rev. Lett. 95, 047402 (2005).

24 A. V. Kimel, A. Kirilyuk, F. Hansteen, R. V. Pisarev, and Th. Rasing, J. Phys. Condens. Matter 19, 043201 (2007).

${ }^{25}$ G. P. Zhang and W. Hübner, Phys. Rev. Lett. 85, 3025 (2000).

26 R. Gomez-Abal, O. Ney, K. Satitkovitchai, and W. Hübner, Phys. Rev. Lett. 92, 227402 (2004).

27 H. Ohno, D. Chiba, F. Matsukura, T. Omiya, E. Abe, T. Dietl, Y. Ohmo, and K. Ohtani, Nature 408, 944 (2000).

28 D. Chiba, M. Yamanouch, F. Matsukura, and H. Ohno, Science 301, 943 (2003).

29 M. Yamanouchi, D. Chiba, F. Matsukura, and H. Ohno,
Nature 428, 539 (2004).

30 S. Koshihara, A. Oiwa, M. Hirasawa, S. Katsumoto, Y. Iye, C. Urano, H. Takagi, and H. Munekata, Phys. Rev. Lett. 78, 4617 (1997).

31 A. Oiwa, Y. Mitsumori, R. Moriya, T. Slupinski, and H. Munekata, Phys. Rev. Lett. 88, 137202 (2002).

32 Y. Mitsumori, A. Oiwa, T. Slupinski, H. Maruki, Y. Kashimura, F. Minami, and H. Munekata, Phys. Rev. B 69, 033203 (2004).

33 A. V. Kimel, G. V. Astakhov, G. M. Schott, A. Kirilyuk, D. R. Yakovlev, G. Karczewski, W. Ossau, G. Schmidt, L. W. Molenkamp, and Th. Rasing, Phys. Rev. Lett. 92, 237203 (2004).

34 J. Wang, I. Cotoros, K. M. Dani, X. Liu, J. K. Furdyna, and D. S. Chemla, Phys. Rev. Lett. 98, 217401 (2007).

35 J. Wang, I. Cotoros, J. Chovan, X. Liu, J. K. Furdyna, I. E. Perakis, and D. S. Chemla, unpublished.

36 T. V. Shahbazyan, I. E. Perakis, and M. E. Raikh, Phys. Rev. Lett. 84, 5896 (2000).

37 J. Fernández-Rossier, C. Piermarocchi, P. Chen, A. H. MacDonald, and L. J. Sham, Phys. Rev. Lett. 93, 127201 (2004).

38 J.Chovan, E.G. Kavousanaki, and I. E. Perakis, Phys. Rev. Lett. 96, 057402 (2006).

39 L. Cywinski and L. J. Sham, Phys. Rev. B 76, 045205 (2007).

40 See e.g. W. Schäfer and M. Wegener, Semiconductor Optics and Transport Phenomena (Springer-Verlag, Berlin, 2002).

41 N. Linder and L. J. Sham, Physica E 2, 412 (1998).

42 G. Lindblad, Commun. Math. Phys. 48, 119 (1976); R. Kosloff and S. A. Rice, J. Chem. Phys. 72, 4591 (1980).

43 L. D. Landau, E. M. Lifshitz, and L. P. Pitaevski, Statistical Physics, Part 2, 3rd ed. (Pergamon Press, Oxford, 1980); L. Landau and E. Lifshitz, Phys. Z. Sov. Union 8, 153 (1935); T. L. Gilbert, Phys. Rev. 100, 1243 (1955).

44 M. Abolfath, T. Jungwirth, J. Brum, and A. H. MacDonald, Phys. Rev. B 63, 054418 (2001).

45 T. Dietl, H. Ohno, and F. Matsukura, Phys. Rev. B 63, 195205 (2001).

${ }^{46}$ U. Welp, V. K. Vlasko-Vlasov, X. Liu, J. K. Furdyna, and T. Wojtowicz, Phys. Rev. Lett. 90, 167206 (2003).

47 K. Hamaya, T. Watanabe,T. Taniyama, A. Oiwa, Y. Kitamoto, and Y. Yamazaki, Phys. Rev. B 74, 045201 (2006).

48 T. Jungwirth, M. Abolfath, J. Sinova, J. Kucera, and A. H. MacDonald, Appl. Phys. Lett. 81, 4029 (2002).

49 I. Zutic, J. Fabian, and S. Das Sarma, Rev. Mod. Phys. 76, 323 (2004).

50 Y. Tserkovnyak, G. A. Fiete, and B. I. Halperin, Appl. Phys. Lett. 84, 5234 (2004).

51 Y. Tserkovnyak, A. Brataas, G. Bauer, and B. I. Halperin, Rev. Mod. Phys. 77, 1375 (2005).

52 A. H. Mitchell, Phys. Rev. 105, 1439 (1957).

53 J. Sinova, T. Jungwirth, X. Liu, Y. Sasaki, J.K. Furdyna, W.A. Atkinson, and A.H. MacDonald, Phys. Rev. B 69, 085209 (2004).

54 M. D. Kapetanakis and I. E. Perakis, Phys. Rev. B 75, 140401(R) (2007); M. D. Kapetanakis, A. Manousaki, and I. E. Perakis, Phys. Rev. B, 73, 174424, (2006); M. D. Kapetanakis and I. E. Perakis, unpublished. 